\title{
A Non-Intrusive Nonlinear Aeroelastic Extension of Loads Packages with Application to a Long Range Transport Aircraft Configuration
}

\author{
Alvaro Cea* and Rafael Palacios ${ }^{\dagger}$ \\ Imperial College, London, United Kingdom
}

\begin{abstract}
A new method for constructing geometrically-nonlinear aeroelastic systems from standard linear models is applied to an industry-level aircraft configuration. The new approach seamlessly integrates with current aeroelastic load packages performing linear analysis based on generic finite-element models (FEMs) and aerodynamic influence coefficient matrices (AICs). We generalize the methodology to incorporate control inputs, find the trimmed aircraft state, or generate gusts disturbances, which can be employed separately or combined to obtain a simplified flight dynamics model for load analysis. An initial study of the aeroelastic response of a long range aircraft is presented. Linear and nonlinear results are introduced in static and dynamic computations of manoeuvres, trim, and gust disturbances. These are compared to commercial software calculations, showing the need for geometrically nonlinear analysis in the production environment of airplanes with ultra high aspect ratio wings.
\end{abstract}

\section{Nomenclature}

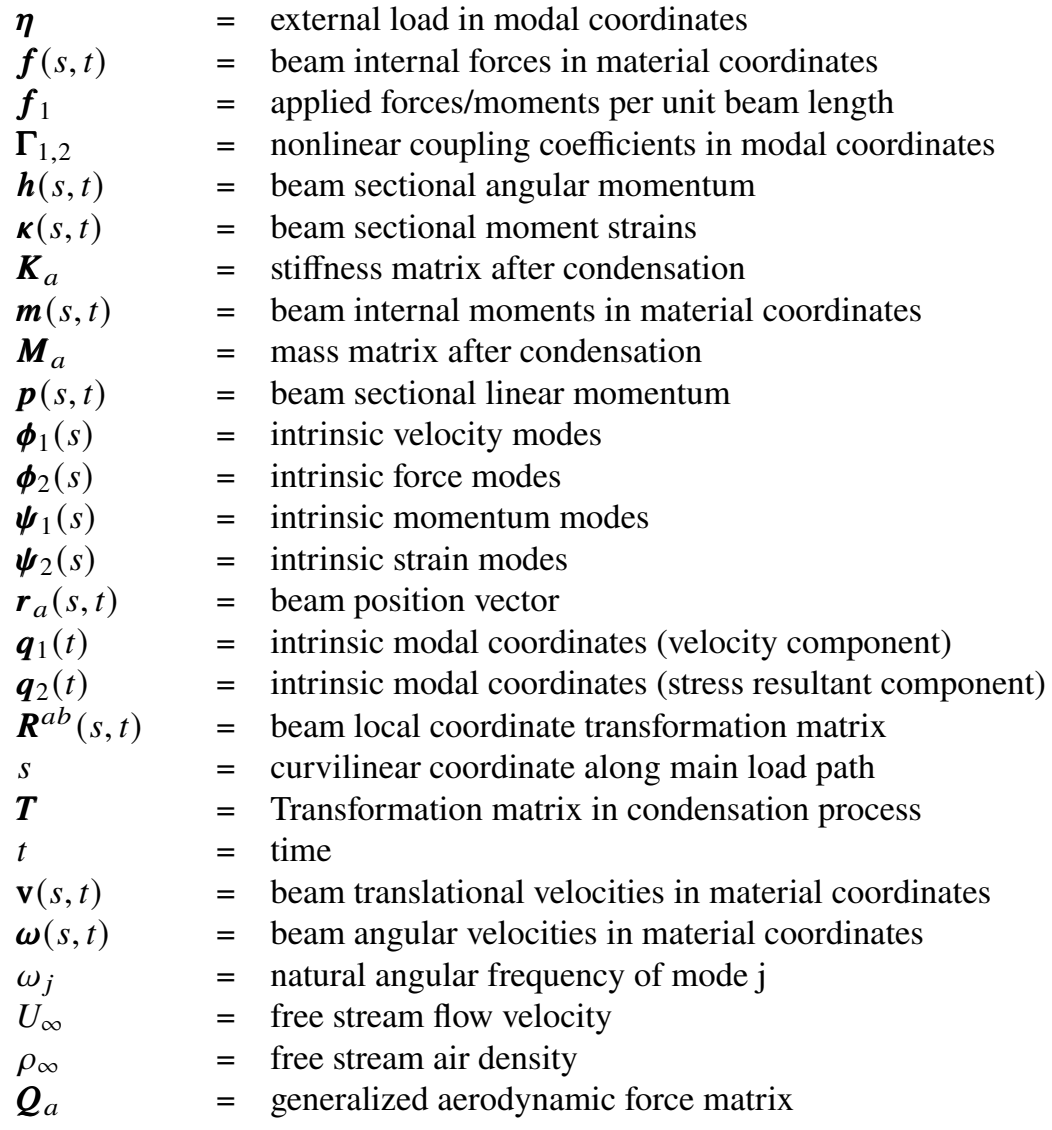

* Research Associate, Department of Aeronautics, South Kensington Campus, SW7 2AZ

${ }^{\dagger}$ Professor of Computational Aeroelasticity, Department of Aeronautics, South Kensington Campus, SW7 2AZ, AIAA Associate Fellow. 


$$
\begin{array}{ll}
\mathcal{A}_{s}, \mathcal{B}_{s}, \mathcal{D}_{s} & =\text { Roger's approximation matrices } \\
\lambda & =\text { aerodynamic states }
\end{array}
$$

\section{Introduction}

Next-generation passenger aircraft will need to meet much stricter emissions and fuel efficiency targets. This will undoubtedly accelerate the push towards increased use of advanced lighter materials to reduce operating weight and configurations with very high aspect ratio wings to reduce induced drag. Aeroelastic effects will thus dominate many aspects of wing design, although higher flexibility may also have positive effects in damping some external disturbances, such as gusts. As a result, airframe design processes may need to be revised [1], and, in particular, geometrical-nonlinear effects may need to be accounted for in stages of the analysis where they are currently neglected. This has already been the case in more radical configurations, such as large solar-powered aircraft [2], where linear analysis yielded non-conservative results as they missed critical nonlinear aeroelastic couplings [3]. While fully-coupled nonlinear aeroelastic simulation is now available [4], certification of a new air vehicle currently requires 100,000 s of load case simulations [5], as it considers manoeuvres and gust loads at different velocities and altitudes, and for a range of mass cases and configurations. Statistical methods may help reduce the total simulation burden, yet there is still a need for computationally-efficient models that provide an acceptable level of fidelity to describe the vehicle dynamics. Furthermore, design of control strategies also requires reduced models, and standard approaches with linear models can fail under large wing deformations [6].

Two main approaches have been considered so far to construct computationally-efficient aeroelastic models with geometrical nonlinearity, namely, descriptions based on beam theory, and model-order reduction methods. Geometrically nonlinear beams have been extensively used to study these problems. See, for instance, the review by [7]. While they have been very useful to understand key physics and explore the design space, they impose substantial limitations when detailed finite-element (FE) models are already available. Extraction of the equivalent beam properties is needed through either a homogenization process [8] or a stick modelling approach [9, 10]. For large structures, it has been shown that either approach can lead to significant errors when compared to nonlinear simulations using built-up finite-element models [11, 12]. Alternatively, system identification approaches have been proposed [13] that capture the nonlinear response of the structure and they are used to construct reduced-order models (ROMs) for fast computations in nonlinear dynamic aeroelasticity [12]. The main issue here lies in the prior calculation of a training database made of a large number of static nonlinear computations, which then acts as the model internal physics in the actual simulations. This approach also needs the full model to be suitable for nonlinear simulations, which could be a real constraint, e.g. on models calibrated against GVT. Another modal-based method has recently been proposed for nonlinear static computations from generic FE models that rely in the calculation of curvature mode shapes, along a reference line, and their subsequent integration to obtain a displacement field in a geometric nonlinear fashion [14]. The flourishing of different methodologies indicates important efforts are being put into finding robust approaches that incorporate nonlinear effects to complex 3D FEMs that were built in a linear fashion and which encompass the knowledge and expertise of organisations, but now require additional features as linear assumptions may no longer be valid.

This work continues the development of an alternative approach [11,15, 16] to study the geometrically nonlinear effects of already existing (linear) industrial-scale aeroelastic models. The method blends the efficiency and accuracy of geometrically-exact 1D-descriptions for problems involving slender components, with the precision of condensation techniques in preserving the full 3D characteristics. The computational framework stands-out for not requiring a prior database of nonlinear computations on the original model, nor having to build equivalent beam models. Instead, the geometric layout of the structure and the condensed stiffness and mass matrices are the only data taken forward to the intrinsic beam equations [17] - that, by construction, take sectional velocities and internal forces as main variables. A feature of this approach is that mass and stiffness properties of the initial configuration require no updating as the model deforms. Furthermore, only quadratic terms of the main variables are needed to capture the complete space of geometric nonlinearities. As for the modelling of the aerodynamic forces, although 2-D airfoil unsteady aerodynamics are still often used for high-aspect-ratio wing aeroelasticity, 3-D effects have been shown to play an important role [18] and will be considered here. The aerodynamic forces are obtained from the Doublet-Lattice Method (DLM) [19, 20], which solves a linearization of the compressible, inviscid, unsteady flow equations in the 3-dimensional domain, and it is commonly used in linear aircraft aeroelastic analysis [21]. Since the structural equations are written in the material frame, the aerodynamic forces naturally appear as follower forces on the structure. It has been shown [16] that key geometrically nonlinear aeroelastic effects (wing inextensionality, geometric stiffening, and follower force effects), can be taken into account despite the linear - i.e. not updating with geometry - aerodynamic matrices. The Unsteady Vortex 
Lattice Method (UVLM) [22] is another potential method for geometrically-nonlinear aeroelastic simulations, as it fully accounts for large deformations both in the structure and the wake. However, it is substantially more computationally demanding and it is limited to incompressible conditions, and therefore not appropriate for the typical flow regimes of commercial aircraft. As the approach is verified, improvements can be easily introduced by amending the aerodynamic matrices from the DLM with experiments and higher fidelity aerodynamics [23, 24]. Moreover, the current description can directly accommodate higher fidelity aerodynamic models, such as 3D panel methods [5] that include thickness effects and CFD-generated aerodynamic influence coefficient matrices [25, 26].

Therefore the full aeroelastic system is formed by coupling the structural Nonlinear Modal Reduced Order Model (NMROM) with the modal generalized aerodynamic forces (GAFs) given by the DLM after transformation to the time domain using Roger's rational function approximation [27]. This was deemed to be a cost effective solution which allows to validate the methods with current industrial practice for loads and to show discrepancies between linear and nonlinear analysis. Subsequently a strategy to trim the aircraft in a longitudinal fashion is introduced, followed by a method to incorporate gust loads with arbitrary shapes in the time domain simulations, so that a full description of the aircraft under operating conditions and geometrically-nonlinear deformations is achieved.

The structure of this paper is as follows. Sec. II presents the main mathematical tools that conform the theoretical backbone behind the computational implementation of FEM INAS, the in-house software on which the calculations shown in Sec. III have been carried out. They encompass a series of standard simulations in flight loads analysis, necessary for sizing the components of the aircraft and ensuring its integrity in flight: the static response under varying angle-of-attack manoeuvres, the nonlinear trimmed configuration, dynamic loads due to gust disturbances, and the assessment of dynamic instability variations under large deformations. Lastly sec. IV]presents the main conclusions drawn from this work and further developments planned for future work.

\section{Aeroelastic Formulation}

The starting point in the formulation is an arbitrarily complex FE model for which structural dynamic condensation techniques are employed to reduce the 3-dimensional components into nodes along all major load paths (a skeleton-like substructure). The resulting linear normal modes (LNMs) of the reduced structure are evaluated in intrinsic variables (velocities and strains) [17] and used as a basis of a Galerkin-projection of the geometrically-nonlinear intrinsic beam equations in modal space [28]. We have generalized previous formulations with a four field description (velocity, momentum, strain, and internal forces) that enables arbitrary reduction techniques and treatment of structures with distributed inertia. The projected equations are solved in time domain with unsteady aerodynamic forces obtained from a doublet lattice. The two-stage structural reduction method has been presented in detail in [11] and the integration of the nonlinear equations of motion with aerodynamic loading in [16]. We build on that framework here to perform static manoeuvres, trim analysis, and dynamic gust responses on a full aircraft configuration. The flight dynamics of the aircraft have been greatly simplified across different methodologies [29-31] in order to be able to analyze the large number of load cases that may occur in flight. The same principle is followed in this work with the aim of adding a geometrically nonlinear solution to the current (linear) environments, with minimum intrusion into the models. Static manoeuvres and the trim state in steady level flight are required in finding the load envelopes that the airframe must withstand; they are calculated as the steady equilibrium of a dynamic simulation, which for the trim condition incorporates rigid body modes, gravity, forces from control devices, and a linear controller to find the state of nonlinear equilibrium. Furthermore, assessment of dynamic gust responses is one of the most important requirements in complying with regulations; they are computed herein in the time domain which allows treatment of arbitrary gust shapes. In what follows we therefore present the methodology to compute trim level flight and atmospheric disturbances, and the basic equations are augmented accordingly to construct the mathematical description that accounts for the different cases encountered in the assessment of loads during flight.

\section{A. Nonlinear modal intrinsic theory}

Consider the solid domain $\Omega$, defining the 3-D structure, and a 1-D sub-domain in which the deformable curve $\Gamma$ defines an internal load path. The points conforming $\Gamma$ either belong to the 3-D structure or are a weighted average of neighboring points. After a finite-element discretization, the linear structural dynamics equations can be written as $\boldsymbol{M} \ddot{\boldsymbol{u}}_{n}+\boldsymbol{K} \boldsymbol{u}_{n}=\boldsymbol{F}$, with $\boldsymbol{M}$ and $\boldsymbol{K}$ the global mass and stiffness matrices, respectively; $\boldsymbol{F}$ the vector of nodal forces/moments and $\boldsymbol{u}_{n}$ the vector of nodal displacements/linear rotations, which includes the nodes of $\Omega$ and $\Gamma$, as the two domains either share nodes or nodes in $\Gamma$ have been linked to $\Omega$ through interpolation elements. Their components are all given with respect to a common inertial reference frame. In order to obtain the condensed model, the degrees of freedom 
(DoF) of the full model are divided into those on active (or master) nodes, located along the load path $\Gamma$; and those of the omitted (or slave) nodes. The displacement vector can therefore be reordered as, $\boldsymbol{u}_{n}=\left(\boldsymbol{u}_{a}^{\top} \boldsymbol{u}_{o}^{\top}\right)^{\top}$, and similarly for the LNMs of the unloaded structure $\boldsymbol{\Phi}=\left[\boldsymbol{\Phi}_{a}^{\top} \boldsymbol{\Phi}_{o}^{\top}\right]^{\top}$. Fig. 1 1 illustrates a typical definition of both 3-D and 1-D domains in a simplified aircraft structure. In the aerodynamic surfaces, the interpolation elements in blue relate the active nodes in the load path to the corresponding cross-sectional nodes of the 3-D structure. On the other hand, the fuselage is modelled as a rigid beam, and its nodes are part of both the full and reduced models. The nodes of the condensed model, in red, represent the discretization of $\Gamma$, where the geometrically nonlinear dynamics will be described.

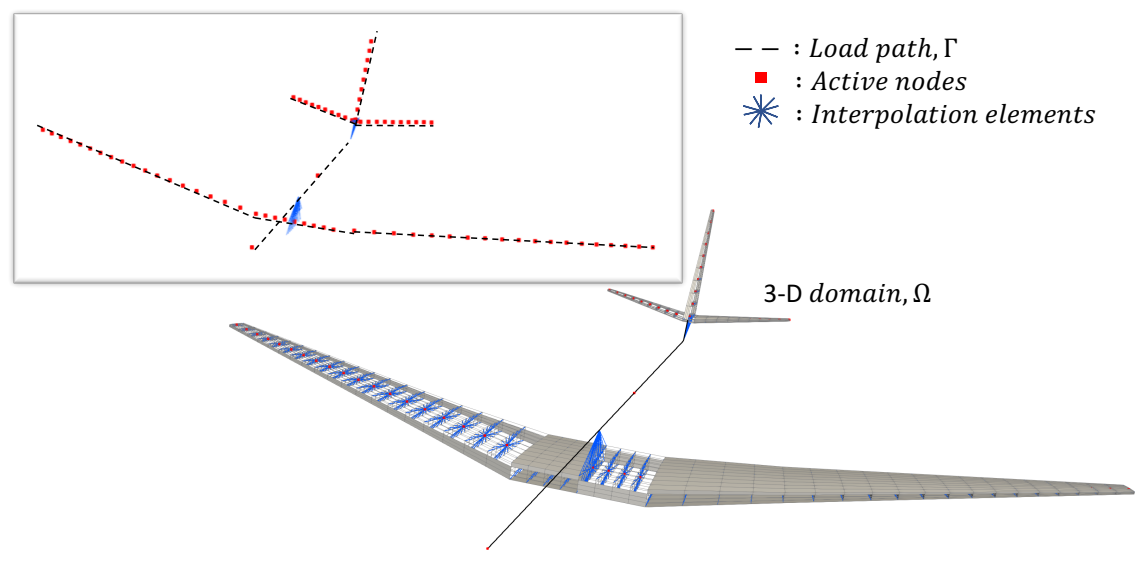

Fig. 1 Structural condensation, 3-D and 1-D domains (aircraft studied in [11])

A linear dependency is now assumed between the omitted and the active degrees of freedom, $\boldsymbol{\Phi}_{o}=\boldsymbol{T}_{o a} \boldsymbol{\Phi}_{a}$, with $\boldsymbol{T}_{o a} \in \mathbb{R}^{o \times a}$ the transformation matrix between both sets. The quality of the transformation is determined by its accuracy to capture a subset of interest of the full-system LNMs. In general, the condensation is dependent on the frequencies and forms a nonlinear eigenvalue problem where each LNM, with natural frequency, $\Omega_{j}$, has one transformation matrix, $\boldsymbol{T}_{o a}$. Different techniques to calculate this matrix, from the most basic Guyan reduction to iterative techniques, have been compared in [16], and are available within the current implementation. After calculation of $\boldsymbol{T}_{\text {oa }}$, the transformation from the active set and the full model is defined as $\boldsymbol{T}=\left[\boldsymbol{I}_{a} \boldsymbol{T}_{o a}^{\top}\right]^{\top}$, with $\boldsymbol{I}_{a}$ the identity matrix of dimension $a$. The condensed stiffness and mass matrix in the restriction to the active set are obtained as $\boldsymbol{K}_{a}=\boldsymbol{T}^{\top} \boldsymbol{K} \boldsymbol{T}$ and $\boldsymbol{M}_{a}=\boldsymbol{T}^{\top} \boldsymbol{M T}$, respectively. The LNMs in the active set are then $\boldsymbol{K}_{a} \boldsymbol{\Phi}_{a}=\boldsymbol{M}_{a} \boldsymbol{\Omega}_{a}^{2} \boldsymbol{\Phi}_{a}$, with $\boldsymbol{\Omega}_{a}$ the diagonal matrix of squared natural frequencies. The level of fidelity preserved in the condensation will depend on how the master nodes are selected and on the ratio between master and omitted nodes. The resulting condensed matrices, $\boldsymbol{K}_{a}$ and $\boldsymbol{M}_{a}$, together with their LNMs, $\left(\Lambda_{a}, \Phi_{a}\right)$, and the spatial coordinates of the active nodes are the only information that is taken forward to the second stage, described next.

The dynamics of a deformable curve along the load path, $\Gamma$, are described using Hodges' intrinsic beam formulation [17]. If $s$ is the curvilinear coordinate along $\Gamma$, we define $\mathrm{v}(s, t) \in \mathbb{R}^{3}$ and $\boldsymbol{\omega}(s, t) \in \mathbb{R}^{3}$ as the components in the local material frame of the inertial translational and rotational velocities, respectively. Their cross-sectional energy conjugates will be the linear and angular momentum, $\boldsymbol{p}(s, t) \in \mathbb{R}^{3}$ and $\boldsymbol{h}(s, t) \in \mathbb{R}^{3}$, respectively. Analogously, we define the sectional internal forces and moments in material coordinates, $\boldsymbol{f}(s, t) \in \mathbb{R}^{3}, \boldsymbol{m}(s, t) \in \mathbb{R}^{3}$, respectively, with their correspondent sectional force and moment strains, $\boldsymbol{\gamma}(s, t) \in \mathbb{R}^{3}$ and $\boldsymbol{\kappa}(s, t) \in \mathbb{R}^{3}$. The external forces and moments, $\boldsymbol{f}_{1}(s, t) \in \mathbb{R}^{6}$, are also given in the deformed material frame, i.e. they are follower forces, which is especially suitable for aerodynamic loading. The state variables are projected into the intrinsic form of the LMNs of the undeformed initial equilibrium equations. Using Einstein's summation convention, the modal projection is defined as,

$$
\begin{array}{ll}
\left(\mathrm{v}^{\top} \boldsymbol{\omega}^{\top}\right)^{\top}=\boldsymbol{\phi}_{1 j}(s) q_{1 j}(t) & \left(\boldsymbol{p}^{\top} \boldsymbol{h}^{\top}\right)^{\top}=\boldsymbol{\psi}_{1 j}(s) q_{1 j}(t) \\
\left(\boldsymbol{f}^{\top} \boldsymbol{m}^{\top}\right)^{\top}=\boldsymbol{\phi}_{2 j}(s) q_{2 j}(t) & \left(\boldsymbol{\gamma}^{\top} \boldsymbol{\kappa}^{\top}\right)^{\top}=\boldsymbol{\psi}_{2 j}(s) q_{2 j}(t)
\end{array}
$$

where $q_{1 j}$ and $q_{2 j}$ are the dual intrinsic modal coordinates for the $j$-th LNM [28], $\boldsymbol{\phi}_{1}$ and $\psi_{1}$ are the respective velocities and momentum mode shapes, respectively, and $\phi_{2}$ and $\psi_{2}$ are the internal forces and strains intrinsic mode shapes. They are approximated here using only the LNMs obtained from the condensation defined above, via a process extensively 
described in [11, 16]. The modal amplitudes are obtained from the nonlinear equations of motion after a Galerkin projection of the intrinsic beam equations, which we write in tensor notation as

$$
\begin{aligned}
& \dot{q}_{1 j}=\delta^{j i} \Omega_{i} q_{2 i}-\Gamma_{1}^{j i k} q_{1 i} q_{1 k}-\Gamma_{2}^{j i k} q_{2 i} q_{2 k}+\eta_{a j}+\eta_{g j}+\eta_{f j} \\
& \dot{q}_{2 j}=-\delta^{j i} \Omega_{i} q_{1 i}+\Gamma_{2}^{i j k} q_{1 i} q_{2 k}
\end{aligned}
$$

where two third-order tensors introduce the gyroscopic, $\Gamma_{1}^{j k l}=\left\langle\phi_{1 j}, \mathcal{L}_{1}\left(\phi_{1 k}\right), \boldsymbol{\psi}_{1 l}\right\rangle$, and strain-force,$\Gamma_{2}^{j k l}=$ $\left\langle\boldsymbol{\phi}_{1 j}, \mathcal{L}_{2}\left(\boldsymbol{\phi}_{2 k}\right), \boldsymbol{\psi}_{2 l}\right\rangle$ nonlinear modal couplings in the dynamic response. Two linear operators $\mathcal{L}_{1}(\boldsymbol{\phi})=[\tilde{\boldsymbol{v}} \mathbf{0} ; \tilde{\boldsymbol{u}} \tilde{\boldsymbol{v}}]$ and $\mathcal{L}_{2}(\boldsymbol{\phi})=[\mathbf{0} \tilde{\boldsymbol{u}} ; \tilde{\boldsymbol{u}} \tilde{\boldsymbol{v}}]$ are defined, for any $\boldsymbol{\phi}=\left(\boldsymbol{u}^{\top} \boldsymbol{v}^{\top}\right)$ and $\tilde{\boldsymbol{\bullet}}$ the skew-symmetric operator. We have also used the inner product in the 1-D domain as $\langle\boldsymbol{\phi}, \boldsymbol{\psi}\rangle=\int_{\Gamma} \boldsymbol{\phi}^{\top} \boldsymbol{\psi} d s$, for any $\boldsymbol{\phi}, \boldsymbol{\psi} \in \mathbb{R}^{6}$, and $\delta^{i j}$ the Kronecker delta. Finally, $\eta_{a j}=\left\langle\boldsymbol{\phi}_{1 j}, \boldsymbol{f}_{a}\right\rangle$ and $\eta_{g j}=\left\langle\boldsymbol{\phi}_{1 j}, \boldsymbol{f}_{g}\right\rangle$ are the modal projection of the external aerodynamic and gravity forces respectively; and $\boldsymbol{\eta}_{f}$ represents the modal component of any other external force in the system. In this paper, we use superindices for matrix/tensor components and subindices for the elements of a column vector. We will refer to equation (2) as the nonlinear modal reduced-order model (NMROM) of the original 3-D structure. It has dimension $2 \times N_{m}$, where $N_{m}$ is the number of LNMs retained from the condensed system. Global positions, $\boldsymbol{r}$, and rotations, $\boldsymbol{R}$, can be recovered either by time-integrations of local velocities, as it is done in rigid body dynamics, or through the integration of strains along the domain $s$, as in the Frenet-Serret formulas of differential geometry [32]. One way is to parameterize the rotation using quaternions $\zeta=\left[\zeta_{0}, \zeta_{1}, \zeta_{2}, \zeta_{3}\right](s, t)=\left[\zeta_{0}, \zeta_{x}\right](s, t)$, as shown in [33]:

$$
\dot{\zeta}=\left[\begin{array}{l}
\dot{\zeta}_{0} \\
\dot{\zeta}_{x}
\end{array}\right]=\left[\begin{array}{c}
-\frac{1}{2} \boldsymbol{\omega}^{\top} \boldsymbol{\zeta}_{x} \\
\frac{1}{2}\left(\zeta_{0} \boldsymbol{\omega}-\tilde{\boldsymbol{\omega}} \zeta_{x}\right)
\end{array}\right]
$$

Given the quaternions the rotation matrix is recovered as,

$$
\boldsymbol{R}=\left(2 \zeta_{x} \otimes \zeta_{x}+\left(\zeta_{0}^{2}-\zeta_{x} \cdot \boldsymbol{\zeta}_{x}\right) \boldsymbol{I}_{3}\right)+2 \zeta_{0} \tilde{\zeta}_{x}
$$

note that the first parenthesis of this equation is the symmetric part of the rotation and the last term the antisymmetric part.

\section{B. Aerodynamic modelling for static and dynamic loads}

For a set of reduced frequencies, $k_{n}$, and a given Mach number, $M_{\infty}$, the DLM yields the modal forces in the frequency domain, $\overline{\boldsymbol{\eta}}$, such that $\overline{\boldsymbol{\eta}}=\frac{1}{2} \rho_{\infty} U_{\infty}^{2} \overline{\boldsymbol{Q}}_{H H} \overline{\boldsymbol{q}}_{0}$. Where $\overline{\boldsymbol{Q}}_{H H}\left(k_{n}, M_{\infty}\right) \in \mathbb{R}^{N_{m} \times N_{m}}$ are the Generalised Aerodynamic Forces (GAFs) that give the forces for a particular set of modal displacements, $\boldsymbol{q}_{0}$, and velocities, $\boldsymbol{q}_{1}$, of the structure. The first step in the construction of an aeroelastic system is the transformation of the aerodynamic forces given by the DLM in frequency domain into the time domain. This is attained by using rational function approximations (RFA), which consist of curve-fitting the GAFs calculated for a range of reduced frequencies and fixed Mach number. Depending on the functional used for the fitting, different techniques have been developed: Roger's approximation using Padé polynomials [27] and a least-square approximation; Karpel's minimum state method [34]; a combination of the two [35], and Chebyshev polynomials [36]. Despite the benefit of methods such as Karpel's in reducing the number states in the final system, it has been reported [37] that methods such as the one proposed by Roger are required in the production engineering environment for their robustness and accuracy in the aeroelastic simulation of loads and gust responses. Therefore Roger's RFA is used to transform the frequency-domain physics into the time-domain and the GAFs are approximated here for a given Mach number as,

$$
\boldsymbol{Q}_{H H}(k)=\mathcal{A}_{0}+(i k) \mathcal{A}_{1}+(i k)^{2} \mathcal{A}_{2}+\sum_{p=1}^{N_{p}} \frac{i k}{\gamma_{p}+i k} \mathcal{A}_{p+2}
$$

In order to complete the RFA the last sum in Eq. (5) defines the aerodynamic states, $\boldsymbol{\lambda}_{p}$ :

$$
\bar{\lambda}_{p}=\frac{i k}{\gamma_{p}+i k} \mathcal{A}_{p+2} \overline{\boldsymbol{q}}_{0}
$$

where $\gamma_{p}$ is the aerodynamic lag associated to $\lambda_{p} \in \mathbb{R}^{N_{m}}$, for $p=1, \ldots, N_{p}$ number of lags in the solution. The selection of these lags can greatly affect the quality of the aeroelastic simulations [38] and gradient-based optimization 
methods are used here to find them. In such cases, the optimizer needs constraints to prevent the lags from collapsing into a single value, or a multipole approach as shown in [39]. In this work, the optimization is carried out in a two-step process: firstly, a non-gradient discrete optimization is performed on the aerodynamic lags with a selected fixed spacing between them; the results are then the initial values of a gradient optimization. Using this approach, lags do not collapse and more optimal values are obtained.

In a similar manner a number $N_{x}$ of control surfaces are modelled through a matrix $\bar{Q}_{H X}\left(k_{n}, M_{\infty}\right)$, from which the control aerodynamic matrices $\mathcal{B}_{i}$ are obtained after a RFA as in Eq. 5. The vector $\boldsymbol{q}_{x}$ comprises the control states of the system, which can go from only one representing the tail-elevator deflection required to trim the aircraft, to an arbitrary number $N_{x}$. Steady terms such as constant angle of attack or side-slip angle, used in the analysis of static manoeuvres, are collected in the state vector $\boldsymbol{\alpha}_{x}$. Their effect is modelled through the matrix $\overline{\boldsymbol{Q}}_{H C}\left(0, M_{\infty}\right)=\boldsymbol{C}_{0}$.

For gust analysis the approach introduced in [40,41] is followed, so the GAF matrix $\bar{Q}_{H J} \in \mathbb{R}^{N_{m} \times N_{j}}$ is obtained from the DLM with $N_{j}$ the number of aerodynamic panels. A RFA is carried out directly on $\bar{Q}_{H J}\left(k_{n}, M_{\infty}\right)$, yielding the matrices $\mathcal{D}_{i}$ and allowing the definition of any upwash function, $\boldsymbol{w}_{g}$, also preventing the spiral nature of the gust problem when approximating directly $\bar{Q}_{H J} \bar{w}_{g j}$. The force in the frequency domain due to a spanwise homogeneous gust is

$$
\bar{\eta}_{g u s t}(\omega)=\frac{1}{2} \rho_{\infty} U_{\infty}^{2} \overline{\boldsymbol{Q}}_{H J}(\omega) \bar{w}_{g j}(\omega)
$$

The upwash, $\bar{w}_{g}$, on a panel $j$ is

$$
\bar{w}_{g j}(\omega)=\boldsymbol{n}_{j} \cdot \frac{\boldsymbol{v}_{g}}{U_{\infty}} e^{-i \omega\left(\boldsymbol{x}_{j}-\boldsymbol{x}_{0}\right) / U_{\infty}}
$$

where $\boldsymbol{n}_{j}$ is the normal to panel $j, \boldsymbol{v}_{g}$ is the vector velocity of the gust (usually in the z-direction for normal gust and in $\mathrm{y}$-direction for lateral gust), $\boldsymbol{x}_{j}$ is the location of the control point in panel $j$, and $\boldsymbol{x}_{0}$ the position of the gust origin. The normal of each panel could be updated as the structure deforms for a complete nonlinear analysis of the gust. However this would entail updating a complex panel geometry for a very short period of time and it is deemed a second order effect here, so no updating of $\boldsymbol{n} \cdot \boldsymbol{v}_{g}$ has been implemented; rather a fully vertical gust is assumed as in [30],

$$
\bar{w}_{g j}(\omega)=\bar{w}_{\text {ref }} \cos \gamma_{j} e^{-i \omega\left(x_{j}-x_{0}\right) / U_{\infty}}
$$

with $\gamma_{j}$ being the dihedral of panel $j$. After application of the Fourier transform the gust upwash in the time domain is put as,

$$
w_{g j}=w_{r e f} \frac{b\left(y_{j}\right)}{2 U_{\infty}} \cos \gamma_{j}\left(1-\cos \left(\frac{\pi U_{\infty}}{H_{g}}\left(t-\frac{x_{0}-x_{j}}{U_{\infty}}\right)\right)\right) H\left(t-t_{0}\right) H\left(t_{1}-t\right)
$$

where and $w_{\text {ref }}$ is defined according to regulations as [42]. $b(y)$ was introduced to change the gust intensity along the spanwise position, and for a standard 1-cos is set to $1 . H_{g}$ is the gust gradient, which is half the gust length $L_{g}$. $H(t)$ is the Heaviside step function with $t_{0}$ and $t_{1}$ defined as $t_{0}=\frac{x_{0}-x_{j}}{U_{\infty}}$ and $t_{1}=\frac{x_{0}-x_{j}}{U_{\infty}}+\frac{U_{\infty}}{L_{g}}$. Note that $\dot{\boldsymbol{w}}_{g}$ and $\ddot{\boldsymbol{w}}_{g}$ are easily calculated from Eq. (10) and they will go directly to Eq. (14) for the gust loads in the full-aeroelastic simulation.

Thus the RFA of $\overline{\boldsymbol{Q}}_{H H}, \overline{\boldsymbol{Q}}_{H X}$ and $\overline{\boldsymbol{Q}}_{H J}$ defines the aerodynamic matrices, $\mathcal{A} \in \mathbb{R}^{N_{m} \times N_{m}}, \mathcal{B} \in \mathbb{R}^{N_{m} \times N_{x}}$ and $\mathcal{D} \in \mathbb{R}^{N_{m} \times N_{j}}$ respectively; and the aerodynamic states, $\boldsymbol{\lambda}_{p} \in \mathbb{R}^{N_{m}}$, for $p=1, \ldots, N_{p}$, with the associated aerodynamic lags or poles, $\gamma_{p}$. Then the total contribution of the aerodynamic forces in the modal space is:

$$
\begin{aligned}
\boldsymbol{\eta}_{a}=\frac{1}{2} \rho_{\infty} U_{\infty}^{2}\left(\mathcal{A}_{0} \boldsymbol{q}_{0}+\frac{c}{2 U_{\infty}} \mathcal{A}_{1} \boldsymbol{q}_{1}+\left(\frac{c}{2 U_{\infty}}\right)^{2} \mathcal{A}_{2} \dot{\boldsymbol{q}}_{1}+\right. \\
+\mathcal{B}_{0} \boldsymbol{q}_{x}+\frac{c}{2 U_{\infty}} \mathcal{B}_{1} \dot{\boldsymbol{q}}_{x}+\left(\frac{c}{2 U_{\infty}}\right)^{2} \mathcal{B}_{2} \ddot{\boldsymbol{q}}_{x}+ \\
+\mathcal{D}_{0} \boldsymbol{w}_{g}+\frac{c}{2 U_{\infty}} \mathcal{D}_{1} \dot{\boldsymbol{w}}_{g}+\left(\frac{c}{2 U_{\infty}}\right)^{2} \mathcal{D}_{2} \ddot{\boldsymbol{w}}_{g}+ \\
\left.+\boldsymbol{C}_{0} \boldsymbol{\alpha}_{x}+\sum_{p=1}^{N_{p}} \lambda_{p}\right)
\end{aligned}
$$


$\boldsymbol{\eta}_{a}$ is accompanied by a system of linear ODEs for the aerodynamic states, which are re-scaled with the dynamic pressure for convenience in the formulation $\left(\lambda_{p} \rightarrow \frac{1}{2} \rho_{\infty} U_{\infty}^{2} \lambda_{p}\right)$, so that

$$
\dot{\lambda}_{p}+\frac{2 U_{\infty} \gamma_{p}}{c} \lambda_{p}=\frac{1}{2} \rho_{\infty} U_{\infty}^{2}\left(\mathcal{A}_{2+p} \boldsymbol{q}_{1}+\mathcal{B}_{2+p} \dot{\boldsymbol{q}}_{x}+\mathcal{D}_{2+p} \dot{\boldsymbol{w}}_{g}\right)
$$

\section{Full aeroelastic integration}

We are now in position to describe the full aeroelastic solution. For $N_{m}$ the number of linear modes selected in the system, $N_{p}$ the number of poles used in the aerodynamics, the final aeroelastic system is built with $2 N_{m}$ nonlinear differential equations that include geometrically-nonlinear modal couplings on the structure; $N_{m}$ integrators to evaluate the modal displacements; $N_{a}=N_{m} \times N_{p}$ linear ODEs for the aerodynamic states; $4 N_{n}$ (four times the total number of nodes) in order to track the rotations through the quaternions $\zeta=\left[\zeta_{0} ; \zeta_{x}\right]$ in Eq. (3), so that gravity forces or other type of dead forces can be rotated to the material frame of reference (it can be the full set of nodes or a reduced set on which the rest are interpolated). Another possibility is to track one node using the quaternions and integrate strains to obtain the rotations for the rest of the nodes. Both techniques have been implemented but we continue the description with the former. The total set of aerodynamic forces conforms the vector $\boldsymbol{\eta}_{a}$ in Eq. (11). On it, $\boldsymbol{w}_{g}$ is a vector with length the number of aerodynamic panels, $N_{j}$, representing any unsteady aerodynamic disturbance (camber effects could also be included in this vector if required); $\boldsymbol{q}_{x}$ the control surfaces in the system, total of $N_{x}$; and $\boldsymbol{\alpha}_{x}$ encompasses the components of any steady manoeuvre, of length $N_{\alpha}$. Inertial forces go into the system after being projected into the modal base $\boldsymbol{\Phi}_{1}$ and rotated to the material frame using the rotation matrix $\boldsymbol{R}$ at each node $l$ written as $\boldsymbol{R}_{l}$ so that the modal gravity component is:

$$
\boldsymbol{\eta}_{g}=\boldsymbol{\Phi}_{1}^{\top} \operatorname{diag}\left(\boldsymbol{R}_{1} \boldsymbol{R}_{1} \ldots \boldsymbol{R}_{N_{n}} \boldsymbol{R}_{N_{n}}\right)^{\top} \boldsymbol{M}_{a} \operatorname{diag}\left(\boldsymbol{I}_{3} \boldsymbol{g}_{0} \mathbf{0}_{3} \ldots N_{n} \ldots \boldsymbol{I}_{3} \boldsymbol{g}_{0} \mathbf{0}_{3}\right)
$$

with $\boldsymbol{M}_{a}$ the condensed mass matrix, $\boldsymbol{g}_{0} \approx\left[\begin{array}{lll}0 & 0 & -9.807\end{array}\right]^{\top}$ the gravity vector at sea level, and $\boldsymbol{I}_{3}, \mathbf{0}_{3}$ the 3 by 3 identity and null matrices respectively. Any other force in the system that does not come from the aerodynamics or the inertia will enter the system after projection as $\boldsymbol{\eta}_{f}$. Putting these definitions together, the complete aeroelastic system is written in tensorial notation as,

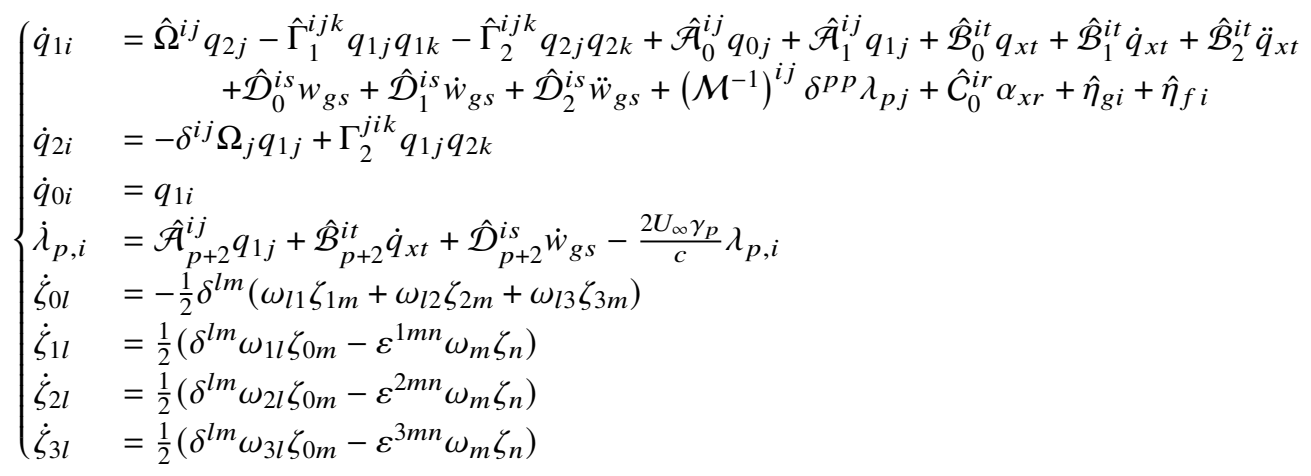

for $i, j, k \in\left\{1, \ldots, N_{m}\right\}, p \in\left\{1, \ldots, N_{p}\right\}, l \in\left\{1, \ldots, N_{n}\right\}, t \in\left\{1, \ldots, N_{x}\right\}, s \in\left\{1, \ldots, N_{j}\right\}, r \in\left\{1, \ldots, N_{\alpha}\right\}$. The Levi-Civita symbol, $\varepsilon_{m n o}$, is introduced for the cross-product in the quaternion terms. The natural frequencies in the system have been written as $\Omega_{j}$ to distinguish them from the angular velocities, $\omega$, and the following re-scaling of the matrices and tensors has been used in order to simplify the notation:

$$
\begin{aligned}
& \mathcal{M}^{i j}=\delta^{i j}-\frac{\rho_{\infty} c^{2}}{8} \mathcal{A}_{2}^{i j} \quad ; \quad \hat{\Omega}^{i j}=\left(\mathcal{M}^{-1}\right)^{i k} \delta^{k j} \Omega_{j} \quad ; \quad\left[\hat{\Gamma}_{1}^{i j k} \hat{\Gamma}_{2}^{i j k}\right]=\left(\mathcal{M}^{-1}\right)^{i h}\left[\Gamma_{1}^{h j k} \Gamma_{2}^{h j k}\right] \\
& {\left[\hat{\mathcal{A}}_{0}^{i j} \hat{\mathcal{B}}_{0}^{i j} \hat{\mathcal{C}}_{0}^{i j} \hat{\mathcal{D}}_{0}^{i j}\right]=\frac{\rho_{\infty} U_{\infty}^{2}}{2}\left(\mathcal{M}^{-1}\right)^{i k}\left[\mathcal{A}_{0}^{k j} \mathcal{B}_{0}^{k j} C_{0}^{k j} \mathcal{D}_{0}^{k j}\right]} \\
& {\left[\hat{\mathcal{A}}_{1}^{i j} \hat{\mathcal{B}}_{1}^{i j} \hat{\mathcal{D}}_{1}^{i j}\right]=\frac{c \rho_{\infty} U_{\infty}}{4}\left(\mathcal{M}^{-1}\right)^{i k}\left[\mathcal{A}_{1}^{k j} \mathcal{B}_{1}^{k j} \mathcal{D}_{1}^{k j}\right]} \\
& {\left[\hat{\mathcal{B}}_{2}^{i j} \hat{\mathcal{D}}_{2}^{i j}\right]=\frac{\rho_{\infty} c^{2}}{8}\left(\mathcal{M}^{-1}\right)^{i k}\left[\mathcal{B}_{2}^{k j} \mathcal{D}_{2}^{k j}\right]} \\
& {\left[\hat{\mathcal{A}}_{p+2}^{i j} \hat{\mathcal{B}}_{p+2}^{i j} \hat{\mathcal{D}}_{p+2}^{i j}\right]=\frac{c \rho_{\infty} U_{\infty}}{4}\left[\mathcal{A}_{p+2}^{k j} \mathcal{B}_{p+2}^{k j} \mathcal{D}_{p+2}^{k j}\right]} \\
& {\left[\hat{\eta}_{g i} \hat{\eta}_{f i}\right]=\left(\mathcal{M}^{-1}\right)^{i j}\left[\eta_{g j} \eta_{f j}\right]}
\end{aligned}
$$


Eq. (14) conforms the aeroelastic NMROM encapsulating the aircraft structure, mass properties, and aerodynamic surfaces.

\section{Nonlinear trimmed state}

While the matrices $\mathcal{B}_{i}$ can bee used to develop a flight dynamics description, here they are only considered to find the trim state of the aircraft, therefore only the tail-elevator control surface is required. For this a series of simplifications are made to reduce complexity and computational times: firstly symmetry of the aircraft around the longitudinal plane is assumed, so that gravity and drag forces are cancelled out by the lift and thrust forces respectively, and the pitching moment is balanced by the tail elevator. A second simplification is carried out in this work as drag and thrust forces are neglected by clamping the vehicle in the $\mathrm{x}$-direction. This assumption is common in load analysis and the rationale behind it is that maximum loads and wing deformations are mostly due to the balance of lift, the pitching moment, and gravity forces. Therefore longitudinal trim of the aircraft is achieved through a dynamic simulation where the rigid body modes for pitching and plunging are included in the analysis and a controller drives the tail elevator angle until equilibrium is achieved. As per the model setting, a point $\boldsymbol{x}_{c}$ is clamped in all directions but the rotation in $y$ and the vertical position in $z \cdot \boldsymbol{x}_{c}$ is chosen to coincide with the center of gravity of the initial configuration, $C g_{0}$, or close to it (it does not matter because the $C g$ position will change as the nonlinear simulation advances). The linear controller outputs the elevator deflection, $q_{x}$, such that a pitching up moment is created when the aircraft is falling, the angle of attack and hence the lift increase, and vice-versa when the lift surpasses the weight. Reaching the steady state in the dynamic simulation will ensure the zero pitching moment condition and having $\boldsymbol{x}_{c}=0$ makes lift due to the wing and elevator and gravity forces cancel out. Note that despite the $C g$ position changing with the nonlinear deformations, the condition of zero pitching moments applies to the whole structure and therefore it is a valid method of finding the nonlinear trim of the aircraft.

\section{System linearization}

A linearization of the aeroelastic nonlinear system is sought so that an efficient approach is generated whereby a steady-state nonlinear solution is firstly obtained, and subsequent analysis can be performed using the linear system. The aeroelastic state variables are grouped as $\boldsymbol{x}=\left[\begin{array}{ll}\boldsymbol{q}_{1}^{\top} & \boldsymbol{q}_{2}^{\top} \boldsymbol{q}_{0}^{\top} \lambda_{p}^{\top} \zeta^{\top}\end{array}\right]^{\top}$ and Eqs. (14) can be written as $\dot{\boldsymbol{x}}=\boldsymbol{g}(\boldsymbol{x})$, where $\boldsymbol{g}$ is a nonlinear function with second order nonlinearities. This aeroelastic system is then linearized around any reference condition $\boldsymbol{x}^{\circ}$, so that $\boldsymbol{x}=\boldsymbol{x}^{\circ}+\boldsymbol{x}^{*}$. Typically the reference is a static equilibrium (i.e., $\boldsymbol{q}_{1}^{\circ}=\mathbf{0}$ and $\lambda_{p}^{\circ}=\mathbf{0}$ for all $p$.). The linear perturbation around the steady state is described as $\dot{\boldsymbol{x}}^{*}=\boldsymbol{G}_{\boldsymbol{x}}\left(\boldsymbol{x}^{\circ}\right) \boldsymbol{x}^{*}+\boldsymbol{\eta}^{*}\left(\boldsymbol{x}^{\circ}\right)$ with $\boldsymbol{G}_{\boldsymbol{x}}$ the Jacobian matrix of the aeroelastic system, $\boldsymbol{G}_{\boldsymbol{x}}=\frac{\partial \boldsymbol{g}}{\partial \boldsymbol{x}}$, a linear function of the steady solution variables, $\boldsymbol{x}^{\circ}$; and $\boldsymbol{\eta}^{*}$ any given perturbation of the external forcing terms.

$$
\boldsymbol{G}_{\boldsymbol{x}}\left(\boldsymbol{x}^{\circ}\right)=\left[\begin{array}{ccccc}
\hat{\mathcal{A}}_{1}-\boldsymbol{L}_{\boldsymbol{q}_{1}}\left(\boldsymbol{q}_{1}^{\circ}\right) & \hat{\boldsymbol{\Omega}}-\boldsymbol{L}_{\boldsymbol{q}_{12}}\left(\boldsymbol{q}_{2}^{\circ}\right) & \hat{\mathcal{A}}_{0} & \boldsymbol{M}^{-1}\left[\boldsymbol{I}_{N_{m}} \cdot N_{p} . . \boldsymbol{I}_{N_{m}}\right] & \boldsymbol{L}_{\boldsymbol{q}_{1} \zeta}\left(\boldsymbol{\zeta}^{\circ}\right) \\
-\boldsymbol{\Omega}+\boldsymbol{L}_{\boldsymbol{q}_{21}}\left(\boldsymbol{q}_{2}^{\circ}\right) & \boldsymbol{L}_{\boldsymbol{q}_{2}}\left(\boldsymbol{q}_{1}^{\circ}\right) & \mathbf{0} & \mathbf{0} & \mathbf{0} \\
\boldsymbol{I}_{N_{m}} & \mathbf{0} & \mathbf{0} & \mathbf{0} & \mathbf{0} \\
\hat{\mathcal{A}}_{P} & \mathbf{0} & \mathbf{0} & -\frac{2 U_{\infty}}{c} \boldsymbol{\gamma}_{p} & \mathbf{0} \\
\boldsymbol{L}_{\zeta \boldsymbol{q}_{1}}\left(\boldsymbol{\zeta}^{\circ}\right) & \mathbf{0} & \mathbf{0} & \mathbf{0} & \boldsymbol{L}_{\zeta}\left(\boldsymbol{q}_{1}^{\circ}\right)
\end{array}\right]
$$

$\gamma_{P}$ and $\mathcal{A}_{P}$ comprise the lag terms and their corresponding aerodynamic matrices:

$$
\hat{\mathcal{A}}_{P}=\left[\begin{array}{c}
\hat{\mathcal{A}}_{3} \\
\vdots \\
\hat{\mathcal{A}}_{2+p}
\end{array}\right] \quad ; \quad \gamma_{P}=\operatorname{diag}\left(\gamma_{1} \boldsymbol{I}_{N_{m}}, \ldots, \gamma_{n_{p}} \boldsymbol{I}_{N_{m}}\right)
$$

The $\boldsymbol{L}_{\boldsymbol{q}} \in \mathbb{R}^{N_{m} \times N_{m}}$ matrices contain the derivatives of quadratic nonlinear couplings corresponding to gyroscopic and stiffness terms:

$$
\begin{aligned}
L_{q_{1}}^{i j} & =\left(\hat{\Gamma}_{1}^{i j k}+\hat{\Gamma}_{1}^{i k j}\right) q_{1 k}^{\circ} \quad ; \quad L_{q_{12}}^{i j}=\left(\hat{\Gamma}_{2}^{i j k}+\hat{\Gamma}_{2}^{i k j}\right) q_{2 k}^{\circ} \\
L_{q_{21}}^{i j} & =\hat{\Gamma}_{2}^{j i k} q_{2 k}^{\circ} \quad ; \quad L_{q_{2}}^{i j}=\hat{\Gamma}_{2}^{k i j} q_{1 k}^{\circ}
\end{aligned}
$$


The $\boldsymbol{L}_{\boldsymbol{q}_{1} \zeta} \in \mathbb{R}^{N_{m} \times 4 N_{n}}$ matrix is the Jacobian of the gravity forces in Eq. 13 combined with any other dead force in the model, $\boldsymbol{f}^{d} \in \mathbb{R}^{6 N_{n}}$, that needs to be rotated to the material frame of reference:

$$
\boldsymbol{L}_{\boldsymbol{q}_{1 \zeta} \zeta}^{l}=\boldsymbol{\Phi}_{1 l}^{\top}\left[\begin{array}{cccccccc}
\boldsymbol{R}_{l, \zeta_{0}}^{\top} & \mathbf{0} & \boldsymbol{R}_{l, \zeta_{1}}^{\top} & \mathbf{0} & \boldsymbol{R}_{l, \zeta_{2}}^{\top} & \mathbf{0} & \boldsymbol{R}_{l, \zeta_{3}}^{\top} & \mathbf{0} \\
\mathbf{0} & \boldsymbol{R}_{l, \zeta_{0}}^{\top} & \mathbf{0} & \boldsymbol{R}_{l, \zeta_{1}}^{\top} & \mathbf{0} & \boldsymbol{R}_{l, \zeta_{2}}^{\top} & \mathbf{0} & \boldsymbol{R}_{l, \zeta_{3}}^{\top}
\end{array}\right]\left[\begin{array}{cccc}
\boldsymbol{f}_{l}^{d} & \mathbf{0} & \mathbf{0} & \mathbf{0} \\
\mathbf{0} & \boldsymbol{f}_{l}^{d} & \mathbf{0} & \mathbf{0} \\
\mathbf{0} & \mathbf{0} & \boldsymbol{f}_{l}^{d} & \mathbf{0} \\
\mathbf{0} & \mathbf{0} & \mathbf{0} & \boldsymbol{f}_{l}^{d}
\end{array}\right]
$$

where $\boldsymbol{L}_{\boldsymbol{q}_{1 \zeta}}^{l} \in \mathbb{R}^{N_{m} \times 4}$ and there is no sum in this case over the index $l$, rather it represents the values of the referred quantity at each particular node. The matrices $\boldsymbol{R}_{, \zeta}$ are found for each node $l$ after differentiating Eq. (4):

$$
\begin{aligned}
& \boldsymbol{R}_{, \zeta_{0}}=\frac{\partial \boldsymbol{R}\left(\zeta^{\circ}\right)}{\partial \zeta_{0}}=2 \zeta_{0}^{\circ} \boldsymbol{I}_{3}+2 \tilde{\zeta}_{x}^{\circ} \\
& \boldsymbol{R}_{, \zeta_{k}}=\frac{\partial \boldsymbol{R}}{\partial \zeta_{x}^{k}}=\frac{\partial}{\partial \zeta_{x}^{k}}\left(2 \zeta_{x}^{i} \zeta_{x}^{j} \boldsymbol{e}_{i} \otimes \boldsymbol{e}_{j}-\delta_{m n} \zeta_{x}^{m} \zeta_{x}^{n} \delta^{i j} \boldsymbol{e}_{i} \otimes \boldsymbol{e}_{j}+2 \zeta_{0}\left(\zeta_{x}^{j} \boldsymbol{e}_{j} \times \boldsymbol{e}_{i}\right) \otimes \boldsymbol{e}_{i}\right) \\
& =\frac{\partial \boldsymbol{R}\left(\zeta^{\circ}\right)}{\partial \zeta_{x}^{k}}=2 \zeta_{x}^{j \circ}\left(\boldsymbol{e}_{k} \otimes \boldsymbol{e}_{j}+\boldsymbol{e}_{j} \otimes \boldsymbol{e}_{k}\right)-2 \zeta_{x}^{k \circ} \delta^{i j} \boldsymbol{e}_{i} \otimes \boldsymbol{e}_{j}+2 \zeta_{0}^{\circ}\left(\boldsymbol{e}_{k} \times \boldsymbol{e}_{i}\right) \otimes \boldsymbol{e}_{i} \quad i, j, k=1,2,3
\end{aligned}
$$

note that these matrices are linear with $\zeta^{\circ}$. Similarly $\boldsymbol{L}_{\zeta \boldsymbol{q}_{1}} \in \mathbb{R}^{4 N_{n} \times N_{m}}$ is written for the node $l$ and the mode $j$,

$$
\boldsymbol{L}_{\zeta \boldsymbol{q}_{1}}^{l j}=\left[\begin{array}{c}
-\frac{1}{2}\left(\boldsymbol{\Phi}_{1 \theta}^{l j}\right)^{\top} \boldsymbol{\zeta}_{x l}^{\circ} \\
\frac{1}{2}\left(\zeta_{0 l}^{\circ} \boldsymbol{\Phi}_{1 \theta}^{l j}-\tilde{\boldsymbol{\Phi}}_{1 \theta}^{l j} \zeta_{x l}^{\circ}\right)
\end{array}\right]
$$

the rest of the entries in the matrix being $\mathbf{0}$ with $\boldsymbol{\Phi}_{1 \theta} \in \mathbb{R}^{3 N_{n} \times N_{m}}$ the angular part of $\boldsymbol{\Phi}_{1} \in \mathbb{R}^{6 N_{n} \times N_{m}}: \boldsymbol{\Phi}_{1 \theta}=\left.\boldsymbol{\Phi}_{1}\right|^{3-6}$. Finally the diagonal matrix $\boldsymbol{L}_{\zeta} \in \mathbb{R}^{4 N_{n} \times 4 N_{n}}$ is,

$$
\boldsymbol{L}_{\zeta}^{l}=\left[\begin{array}{cc}
0 & -\frac{1}{2} \boldsymbol{\Phi}_{1 \theta}^{l} \boldsymbol{q}_{1}^{\circ} \\
\frac{1}{2}\left(\boldsymbol{\Phi}_{1 \theta}^{l} \boldsymbol{q}_{1}^{\circ}\right)^{\top} & -\frac{1}{2} \boldsymbol{\Phi}_{1 \theta}^{l} \boldsymbol{q}_{1}^{\circ} \boldsymbol{I}_{3}
\end{array}\right]
$$

As previously mentioned, if the nonlinear reference condition comes from a static equilibrium, $\boldsymbol{q}_{1}^{\circ}=\mathbf{0}$, and $\boldsymbol{L}_{\boldsymbol{q}_{1}}=\boldsymbol{L}_{\boldsymbol{q}_{2}}=$ $\boldsymbol{L}_{\zeta}=\mathbf{0}$. 


\section{Numerical Results}

The mathematical theory partially described above has been implemented into the software FEM $_{4}$ INAS (FiniteElement-Models for Intrinsic Nonlinear Aeroelastic Simulations), first introduced in [16]. It is an aeroelastic toolbox written and parallelised in Python, which acts as a post-processor of MSC Nastran, or other packages for loads, such that it enhances arbitrary FEMs built for aeroelastic analysis with geometric nonlinear effects, flight dynamics and linearized state-space solutions about nonlinear trim points. We demonstrate the capabilities of $\mathrm{FEM}_{4}$ INAS in a complex aircraft configuration built by Airbus for research and collaboration. First of all an overview is given of the main characteristics of this aircraft, showing that the reduced model that goes into the nonlinear system of equations is actually a very accurate representation of the original model. Numerical results begin with static equilibrium calculations for different angles of attack, showing a good agreement with MSC Nastran for small deformations and disparities coming the nonlinear effects for larger AoA; then the trim solution is tested for a range of load cases, proving a reduction of angle of attack in the equilibrium condition with respect to the linear analysis as the loads increase; the dynamic response for different gust shapes is compared under linear and nonlinear settings, which points towards the idea that geometrically nonlinear analysis may be even more important in dynamic simulations; the nonlinear flutter speed is investigated for rising angles of attack that induce large deformations, and the result is a significant reduction in the flutter speed when compared to the linear solution. Finally the open-loop response of the aircraft is shown for a combination of trim and gust loads.

\section{A. Reference aircraft description: XRF1 model}

The studies presented in this paper are based on a reference configuration developed to industry standards by Airbus as part of the eXternal Research Forum (XRF), from which the aircraft takes his name, XRF1. The aircraft represents a long range wide body transport airplane and has been used as a research platform for collaboration between the company, universities and research institutions. For this work the model has been modified and contains a wing tip extension that makes the overall aspect ratio of the wing higher and can be used as an aeroelastic hinge device for load alleviation, although this is not demonstrated herein and the whole mechanism remains attached. Since the GFEM itself has been slightly modified the baseline configuration is called XRF1-IC (for Imperial College). Fig. 2 shows the full aeroelastic model split up into the structural, mass and aerodynamic components.

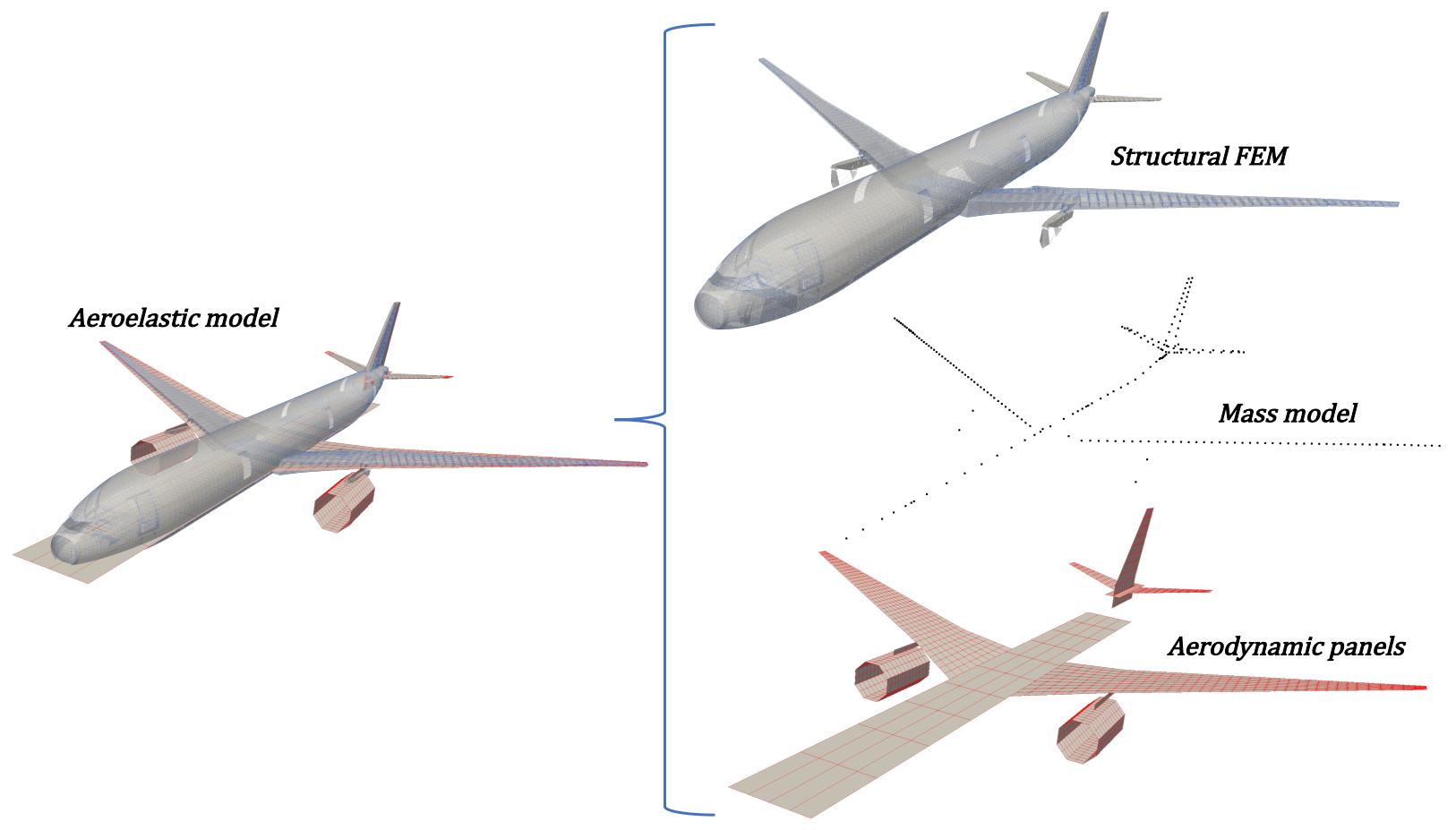

Fig. 2 XRF1-IC aeroelastic subcomponents

The FE model contains a total of around 177400 nodes, which are condensed into 176 active nodes along the 
reference load axes through interpolation elements (RBE3s with the UM option as described in [31]). A Guyan or static condensation approach is used for the reduction, which is equivalent to a dynamic condensation when the mass model is given as discrete elements at the condensation points, as is the case here - for distributed mass elements other dynamic condensation techniques might give better results as shown in [16]. Among the FE elements conforming the model there are around $\sim 500$ spring, or 0-dimension, elements; $~ 57,000$ beam elements, $\sim 55,500$ CQUAD4 shell elements, $\sim$ 3, 800 CTRIA3 shell elements; $~ 800$ rigid elements. As for the mass configurations, the one utilised in this study is the estimated cruise reference mass, which amounts to a total of $\sim 188,500 \mathrm{Kg}$. The aerodynamic model contains $\sim 1,500$ aerodynamic panels.

While there might be other methods for working with these large models in an efficient manner and incorporating geometrically nonlinear effects, one of the major advantages of the current methodology is that prior nonlinear calculations are not required to find the ROM, nor equivalent designs built that could lose some details of the original model. The only operation is a condensation step where the full aircraft model is reduced into the major load-paths. This is already a common practise in the aeroelastic industrial environment and the reduced model of the XRF1 was provided too. The strength of the approach can be seen by looking at the natural frequencies of the full and reduced models, shown in Table 1 normalised with the first natural frequency. An approximate categorisation of the LNMs is also included.

Table 1 Normalized natural frequencies of the XRF1-IC clamped model

\begin{tabular}{cccc}
\hline \hline Mode Type & $\omega_{\text {f ull }}$ & $\omega_{\text {condensation }}$ & Error (\%) \\
First out-of-plane wing bending (1) & 1.0 & 1.0 & 0.0001 \\
First out-of-plane wing bending (2) & 1.04948 & 1.04949 & 0.0004 \\
First fuselage bending (3) & 1.4285 & 1.4288 & 0.025 \\
Fuselage lateral bending + wing out-of-plane bending (4) & 1.5093 & 1.5099 & 0.038 \\
Wing and pylon lateral roll bending (5) & 2.1449 & 2.1449 & 0.0007 \\
$\vdots$ & & & \\
First in-plane wing bending (13) & 3.156 & 3.155 & 0.03 \\
Second in-plane wing bending (14) & 3.25655 & 3.2566 & 0.0016 \\
$\vdots$ & & & \\
First wing torsion (28) & 7.675 & 7.676 & 0.01 \\
Second wing torsion (29) & 7.7368 & 7.7366 & 0.002 \\
\hline \hline
\end{tabular}

The preservation of fidelity in the frequencies is remarkable: a maximum error of $0.038 \%$ in the first 10 modes, 0.35 $\%$ in the first 25 and $3.21 \%$ in the first 50. In comparison Riso et al [10] have obtained an equivalent beam model of the XRF1 with a FEM to stick approach which incurs in errors of up to $10.3 \%$ only within the first 10 modes.

From the point of view of the aerodynamics, 8 poles are used in the rational function approximation of the frequency domain calculations obtained from the DLM. An excellent fit is attained with the optimised approximation of the poles as shown in Fig. 3. It is remarkable that if in the cantilever example shown in [11] a good approximation could be found without optimising the poles just by increasing their number, in this more complex case an accurate fit is not obtained unless they are optimised, as when the number of poles is increased, unwanted oscillations occur due to over-fitting. 

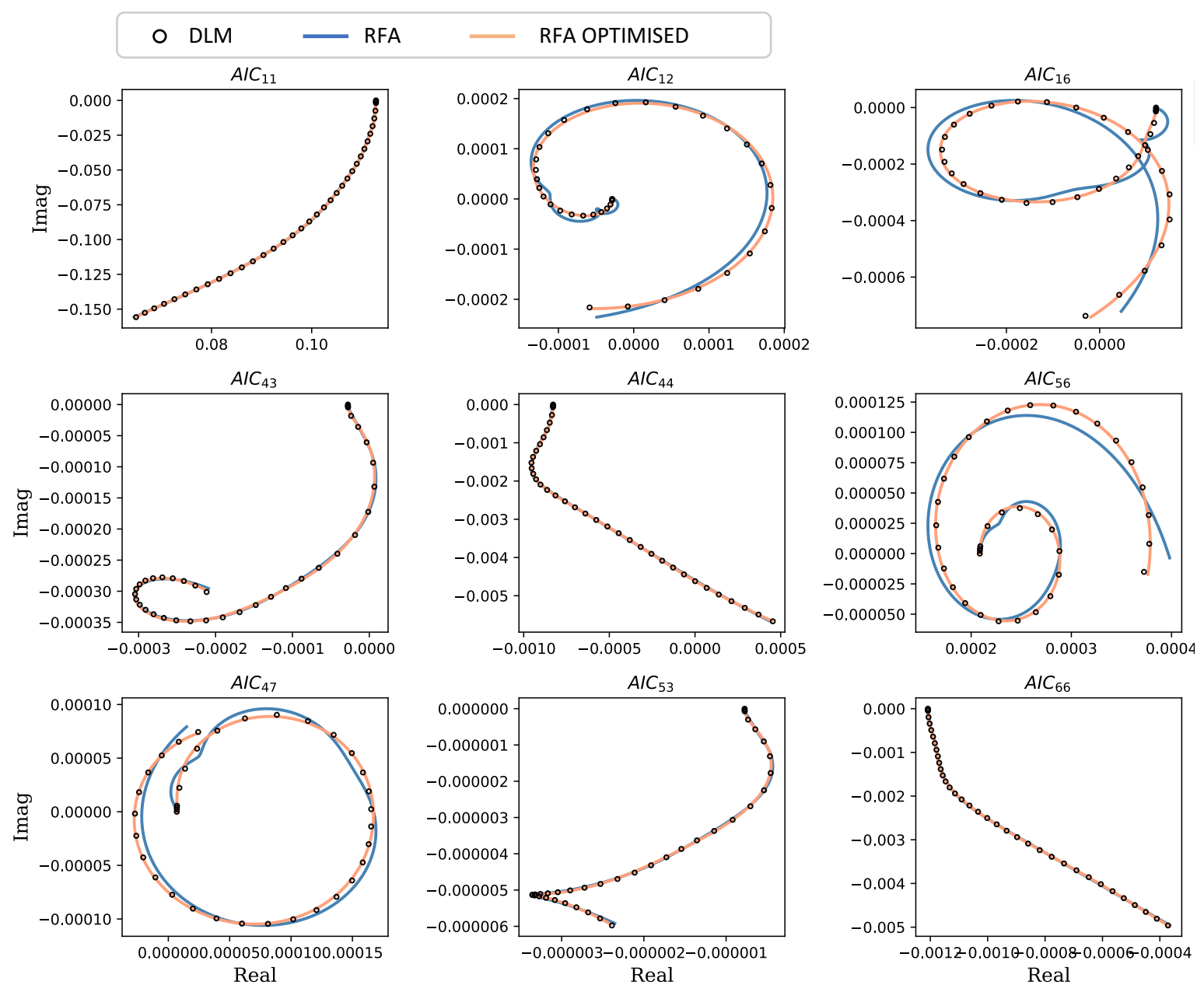

Fig. 3 Static response of aircraft condensation points for angle of attack variations

\section{B. Static manoeuvres}

The aircraft is clamped close to the wing-box. The steady response under an angle of attack aerodynamic loading is calculated for a dynamic pressure of $q_{\infty}=24500 \mathrm{~Pa}$ and 0 Mach number conditions, both using MSC Nastran static linear solvers (SOL 144 on the full model, i.e. without Guyan reduction), and solving the dynamic equilibrium of a NMROM built in FEM 4 INAS, Eq. (14) with $\alpha_{x}$ containing only the particular angle of attack. Fig 4 shows the 3-D response of the condensed structure along the load paths for $A o A=\left[1^{\circ}, 3^{\circ}, 7^{\circ}, 11^{\circ}, 14^{\circ}\right]$. A good match is found for small angles and, for larger angles, nonlinear effects, such as wing shortening and follower forces, are visible.

The NMROM for these results is constructed with 70 structural modes, after a convergence analysis was carried out taking a solution with 100 modes as the reference and using the $L_{2}$ norm on the wing displacements to define the error metric: $e r r=\left\|u_{\text {wing }}^{100}-u_{\text {wing }}^{N_{m}}\right\| /\left\|u_{\text {wing }}^{100}\right\|$. Fig. 5 shows the error between approximations as the angle of attack augments, i.e. for an increasing level of wing deformations. A shaded blue region represents errors where differences are small enough that solutions can be considered good for the purposes of this work (below 3-4 ${ }^{\circ}$, an almost identical response has been found between MSC Nastran linear solution and our solvers). Note that the equilibrium condition itself in the NMROM comes from a dynamic simulation where small, undamped oscillations may affect decimal places unnoticeable in the overall structural state but having small effects on the error convergence. 


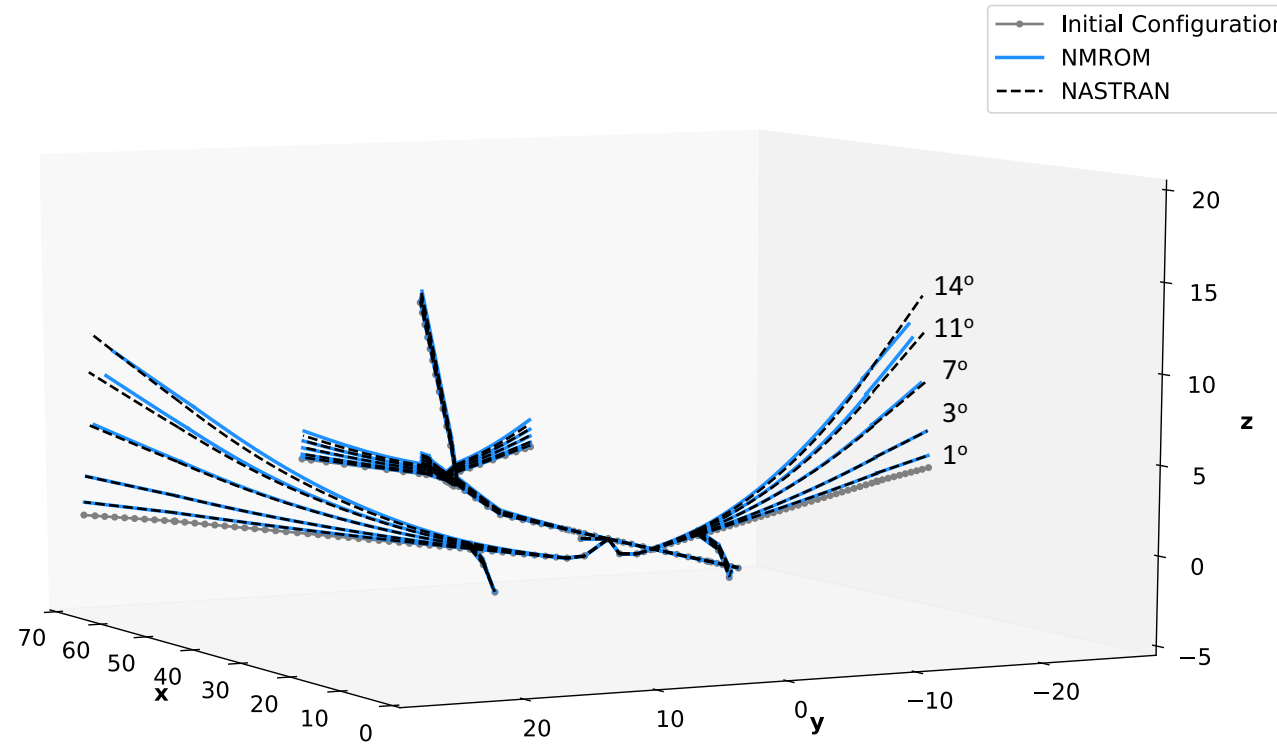

Fig. 4 Static response of aircraft condensation points for angle of attack variations, $q_{\infty}=24500 \mathrm{~Pa}$ and $M_{\infty}=0$

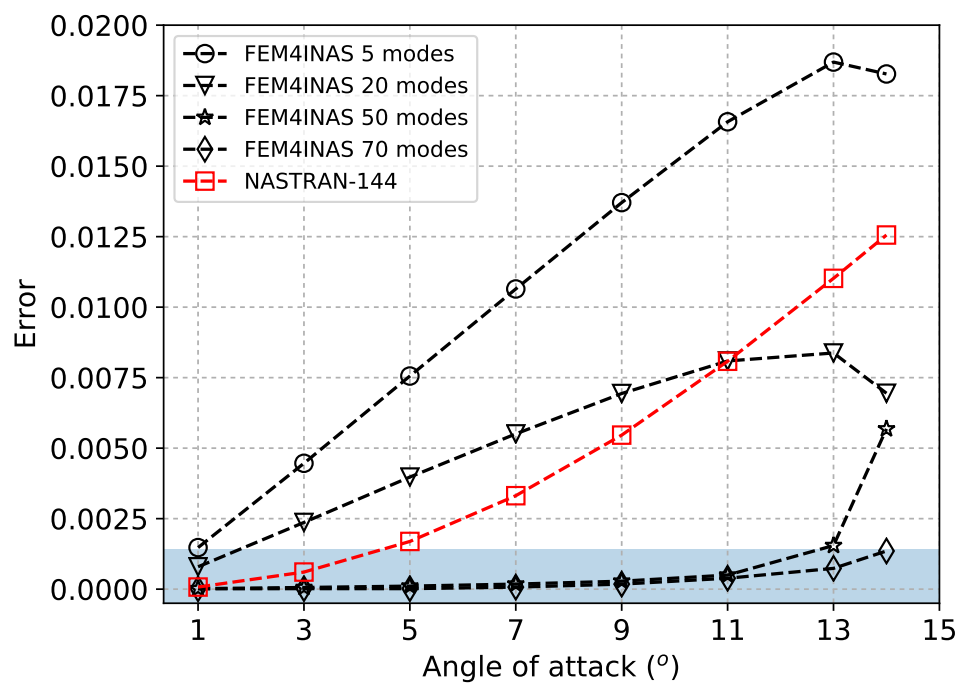

Fig. 5 Error convergence in the XRF1 static response under AoA (NMRON with 100 structural modes used as the reference)

We have taken a sectional view of the wing condensation points in Fig. 6 by showing the normalised wing vertical position versus a metric of $x$ and $y, \sqrt{x_{a}^{2}+y_{a}^{2}}$. It can be seen that up to around $5^{\circ}$, which represents a vertical displacement of about $10 \%$ of the wing semi-span, there is very good agreement between MSC Nastran and FEM $_{4}$ INAS results and, from there onwards, differences become more prominent.

In order to better see the nonlinear effects, the wing tip $x, y, z$ deflections are plot in Fig. 7 after normalised ( $\hat{\bullet})$ with the wing semi-span. If the linear evolution of MSC Nastran is clear, the NMROM results show larger $x$ and $y$ components, while the $z$ component decreases with respect to the linear results. This is exactly what is expected as the structure deforms and aerodynamic forces are no longer pointing only in the $z$-direction. 


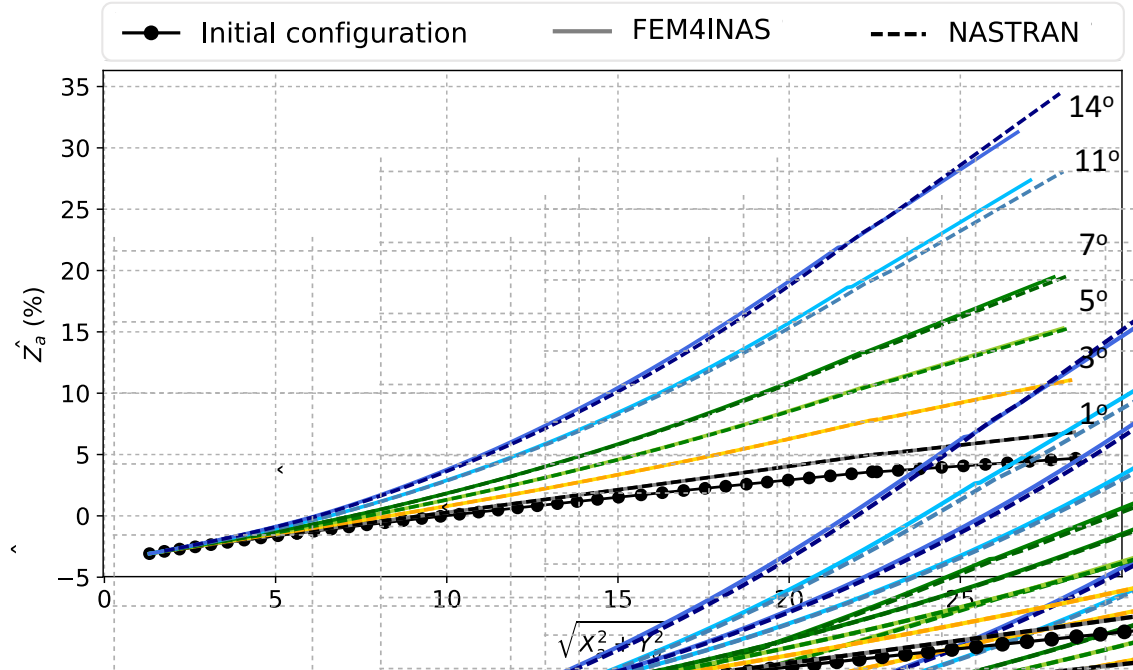

Fig. 6 Sectional view of the aircraft right-wing
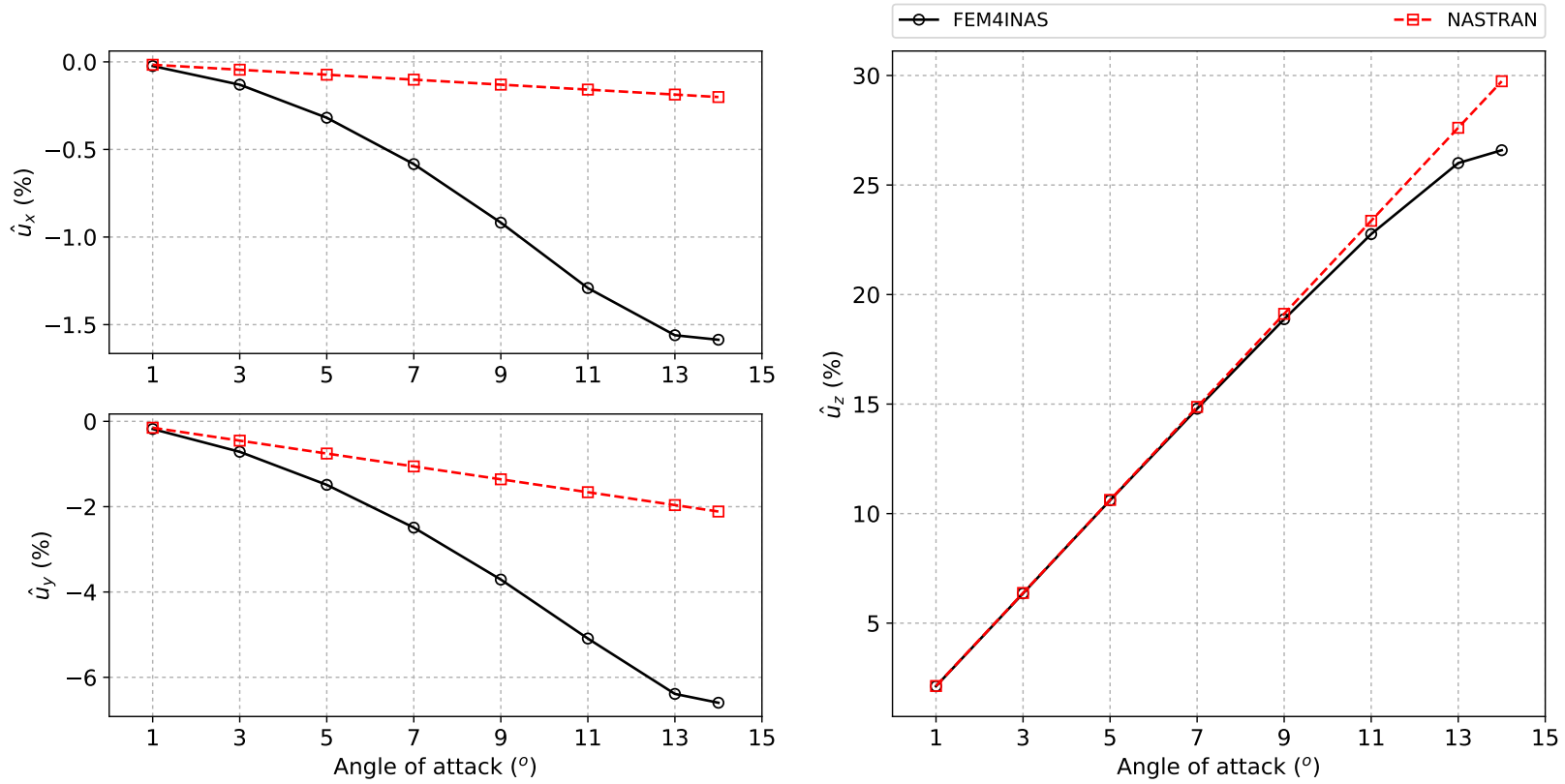

Fig. 7 Aircraft wing-tip deflections in \% of semi-span length VS angle of attack

\section{Nonlinear aeroelastic trim}

Next in the analysis we consider the trim state of the aircraft for accelerations going from $1 \mathrm{~g}$ to $3.5 \mathrm{~g}$. Even though the upper limit for design is $2.5 \mathrm{~g}$, this is a conventional aircraft not conceived to undergo very large deflections so that design limits have been surpassed to display strong nonlinearities, comparable to those expected within the design limits in next generation aircraft with ultra high aspect ratio wings and very flexible structures. Thus the aircraft trimming configurations are shown in Fig. 8 for 1g, $2 \mathrm{~g}$ and $3.5 \mathrm{~g}$ loadings. The comparison is made on the reduced model between MSC Nastran linear solution on the full model (SOL 144) and a NMROM built with 70 structural modes -based on the convergence exercise above. The flight conditions are $q_{\infty}=13313.5 \mathrm{~Pa}$ and $M_{\infty}=0$ Note that in this simulation the aircraft is not clamped but includes the rigid body modes corresponding to pitching and plunging, all of which is automatically taken care of in our implementation. 


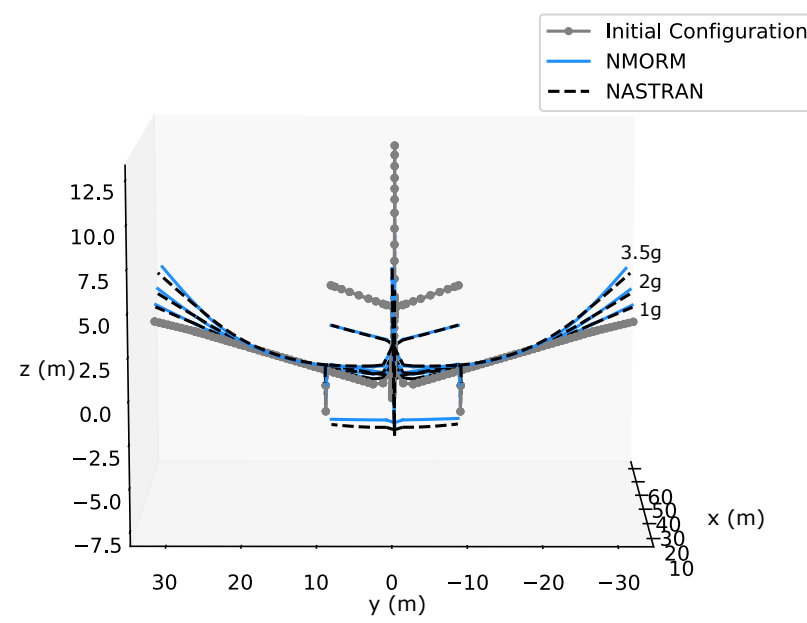

(a) Front view

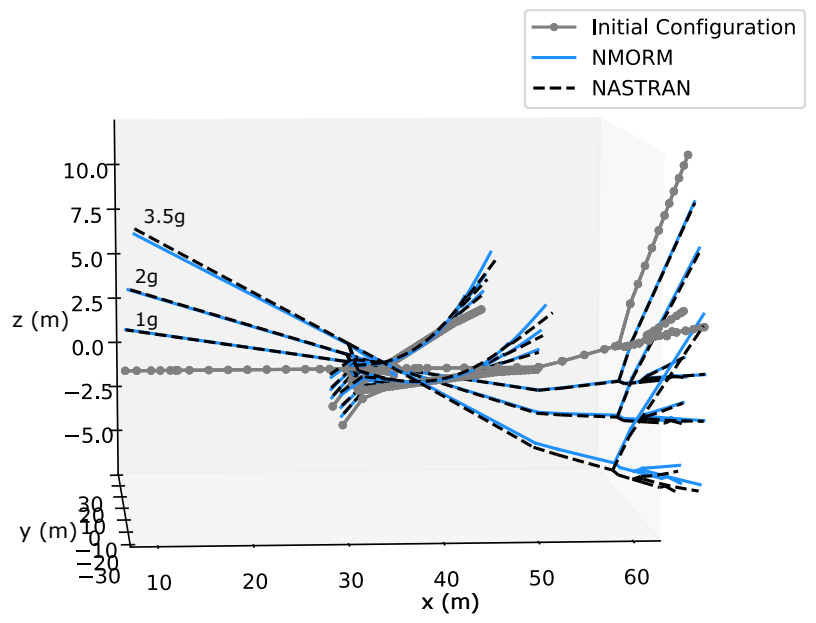

(b) Side view

Fig. 8 3D trimmed configuration of aircraft under increasing gravity accelerations

Fig. 8 shows that nonlinear effects are negligible at $1 \mathrm{~g}$ and some discrepancies appear at $2 \mathrm{~g}$ which are intensified at 3.5g. Perhaps the differences between the linear and nonlinear analysis are better illustrated by showing the key figures in controlling the overall lift and moment of the aircraft, i.e. the AoA and elevator angle at the trim condition. These are shown in Fig. 9 with the added computation of a new flight condition with $q_{\infty}=7076 \mathrm{~Pa}$ and $M_{\infty}=0$.

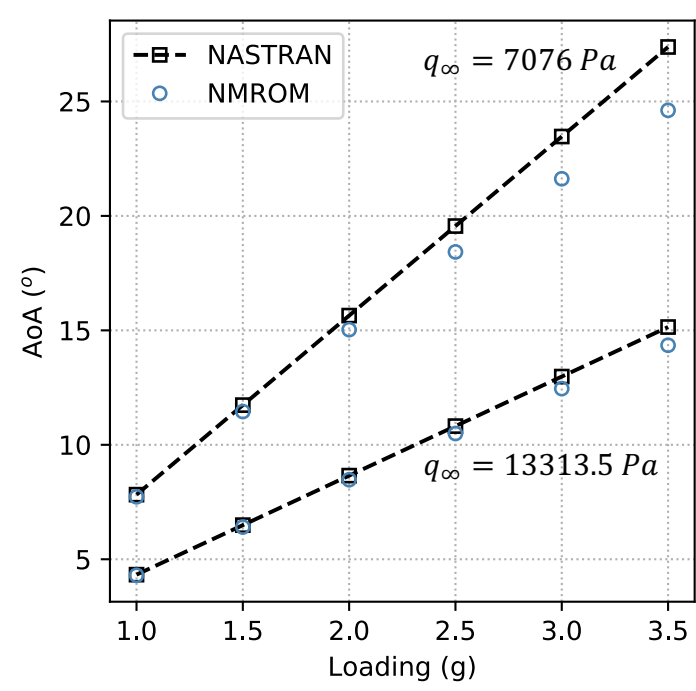

(a) AoA

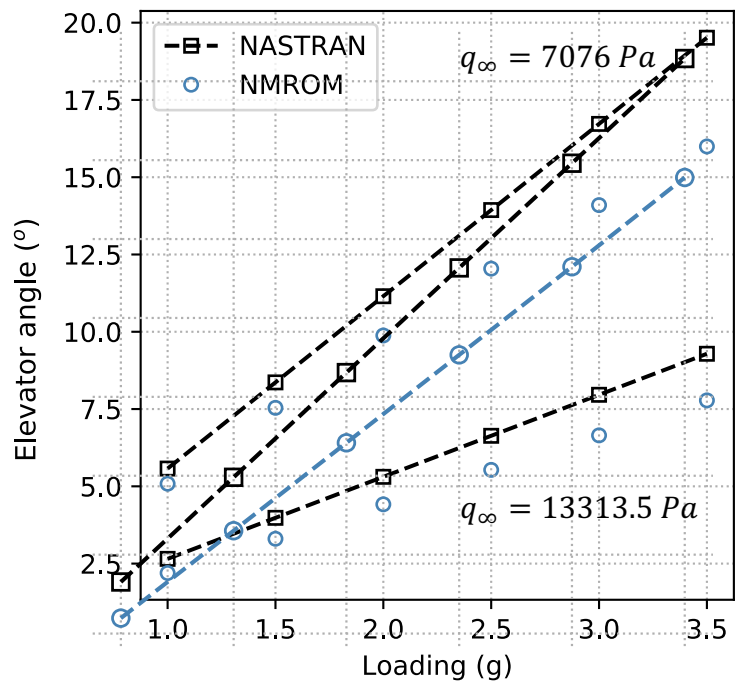

(b) Elevator deflection

Fig. 9 Aircraft angle of attack and elevator deflection

We see a very good agreement for small loadings and a slight decrease in the AoA for higher loadings with respect to the linear computations. Differences are more significant in the elevator angle, specially for higher AoA. This might be due to the correlation between the decrease in AoA and elevator angle, and also the effect of follower forces in creating the required arm moments. Because the trim condition is achieved through a dynamic simulation that converges to a steady state, we can show some figures of merit in the time-domain simulations such as angle of attack, tail elevator deflection or the z-component of the reference point, $x_{c}$, that the linear controller aims to put to zero. These are shown in Fig. 10 


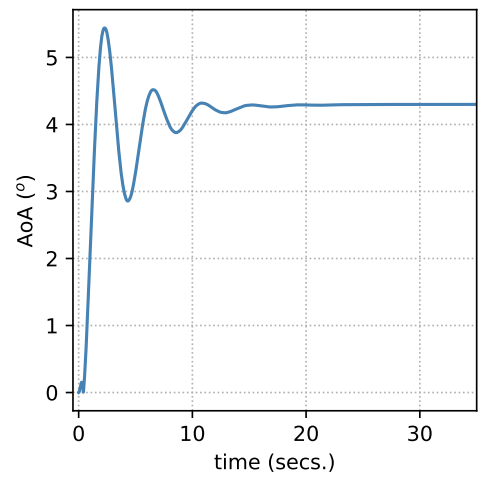

(a) Angle of attack

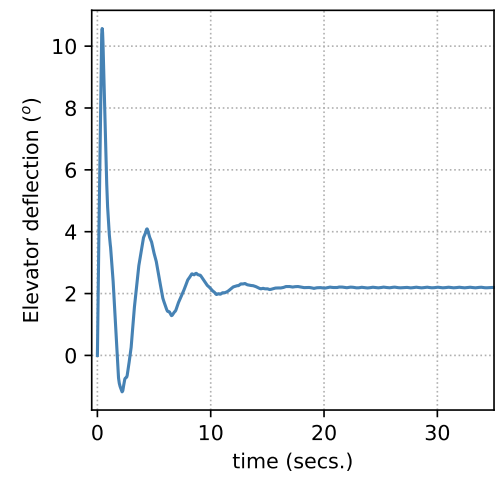

(b) Elevator angle

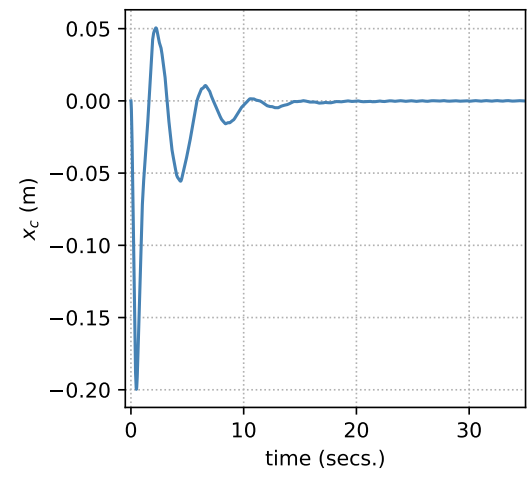

(c) Z-position

Fig. 10 Evolution in time of key variables in the $1 \mathrm{~g}$ flight trim simulation, $q_{\infty}=13313.5, M_{\infty}=0$

\section{Gust response}

If the previous two exercises were aimed at predicting the aeroelastic static equilibrium of the aircraft, we set out now to study its dynamic response under linear and nonlinear conditions. It is important for certification to analyse the effect of atmospheric disturbances in the aircraft loads. Control strategies and load alleviation techniques do also rely on having a good description of the aircraft dynamics in flight. A simple representation of atmospheric disturbances is the 1-cos gust type of shape, constant along the wing span and travelling in the x-direction with that particular shape, as described in Eq. [10, and where $w_{r e f}$ is defined according to regulations as [42],

$$
w_{\text {ref }}=w_{g_{0}}\left(\frac{H}{106.17}\right)^{1 / 6}
$$

with $H$ given in meters. $w_{g_{0}}$ is also tabulated as a function of the altitude, although it will be used here as a scaling factor to clearly differentiate the boundaries between linear and nonlinear analysis. This type of gust will be used to validate the approach by comparing with the linear results from MSC Nastran, which performs the calculations in the frequency domain and converts them to the time domain via an inverse Fourier transform. Arbitrary gust shapes, even random type of signals, can be input within the current framework too, so this will be demonstrated with across the span shapes illustrated in Fig. 11 The travelling wave in the $\mathrm{x}$-direction remains a cosine shape for convenience, but any other type could have been used.

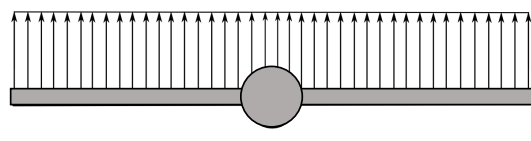

a) Constant

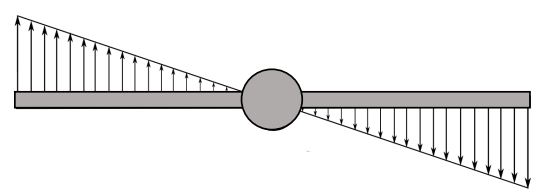

(b) Asymmetric linear

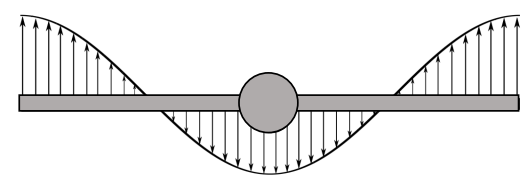

(c) DARPA

Fig. 11 Span-wise gust shapes

From these profiles, a constant shape represents the classical 1-cos disturbance, the antisymmetric linear is good for assessing rolling dynamics and the DARPA shape gust features a cosine shape along the span. Hence the gust profile in Eq. (10) is used with $b(y)$ catering for the shapes across the span just described. We begin validating the gust input system as well as the the aeroelastic dynamic solvers by comparing the dynamic response of the clamped aircraft for a range of 1-cos gusts with small intensities. As per CS-25 and FAR regulations, gust lengths must be within the range (in meters) of $L_{g} \in[18,214]$. Based on this, four types of gusts are selected, with the following gust lengths, [1:18 m;2:67 m;3:116 m; $4: 214 \mathrm{~m}$ ], gust intensity of $w_{g_{0}}=0.01$ in Eq. 23. The flow conditions are air speed $U_{\infty}=185 \mathrm{~m} / \mathrm{s}$, air density of $\rho_{\infty}=0.778 \mathrm{Kg} / \mathrm{m}^{3}$ and the Mach number is set to 0 , so no compressible effects are 
accounted for. A very good match is found considering the differences in the approaches. The wing-tip normalised displacements are shown in Fig. 12 .
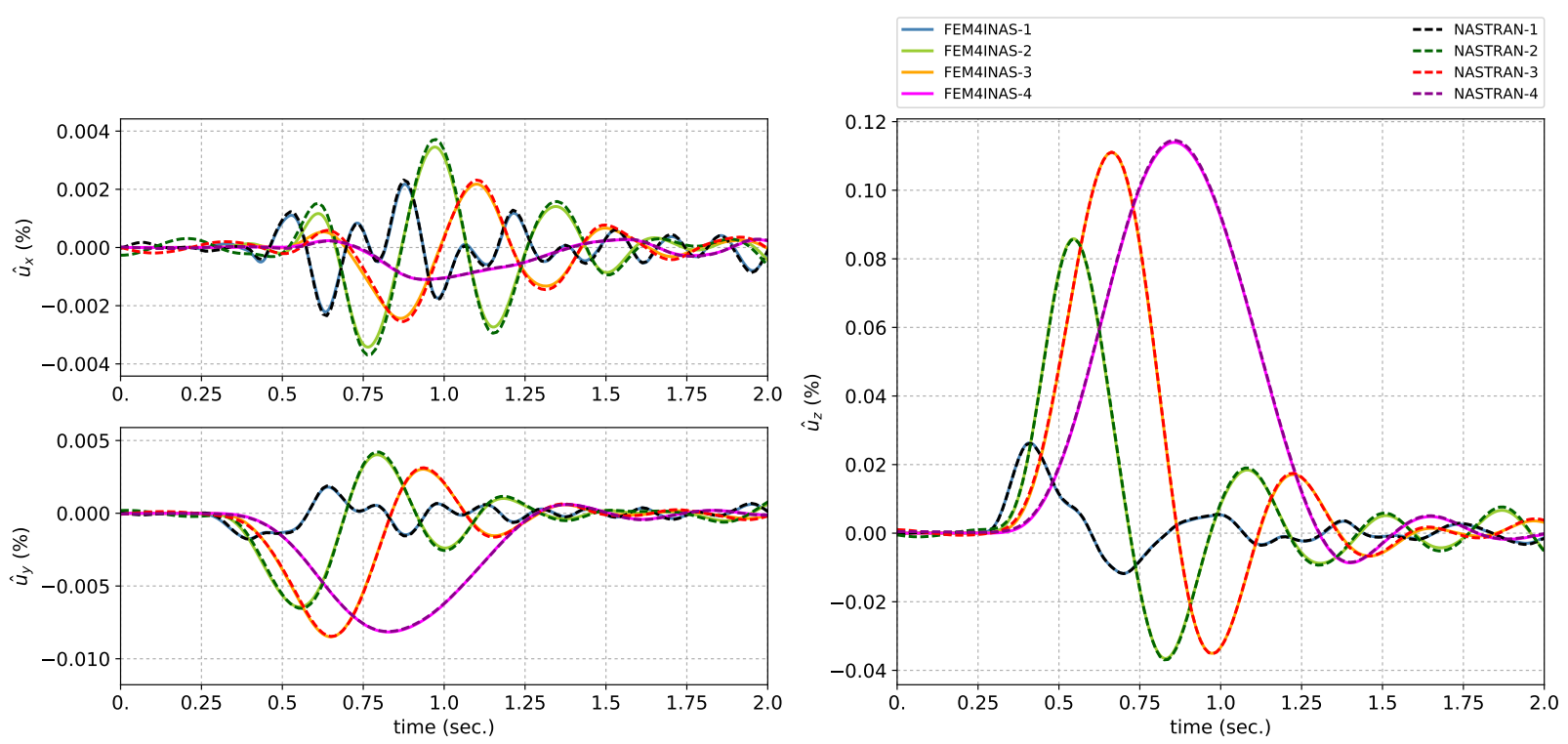

Fig. 12 Wing-tip dynamic response for low intensity gust at Mach 0

The gust intensity is then increased so that nonlinear effects become relevant. The gust intensity is set to $w_{g 0}=3$ for gust number 2 and to $w_{g 0}=2$ for gust number 4 and results are presented in Fig 13 As expected larger discrepancies are seen in the $x$ and $y$ directions and a slightly smaller component appears in the $z$ direction of the nonlinear computations. It is also apparent a frequency shifting do occur specially under gust 2 , which has been reported previously in geometrically nonlinear dynamic studies of simpler problems [12, 43].
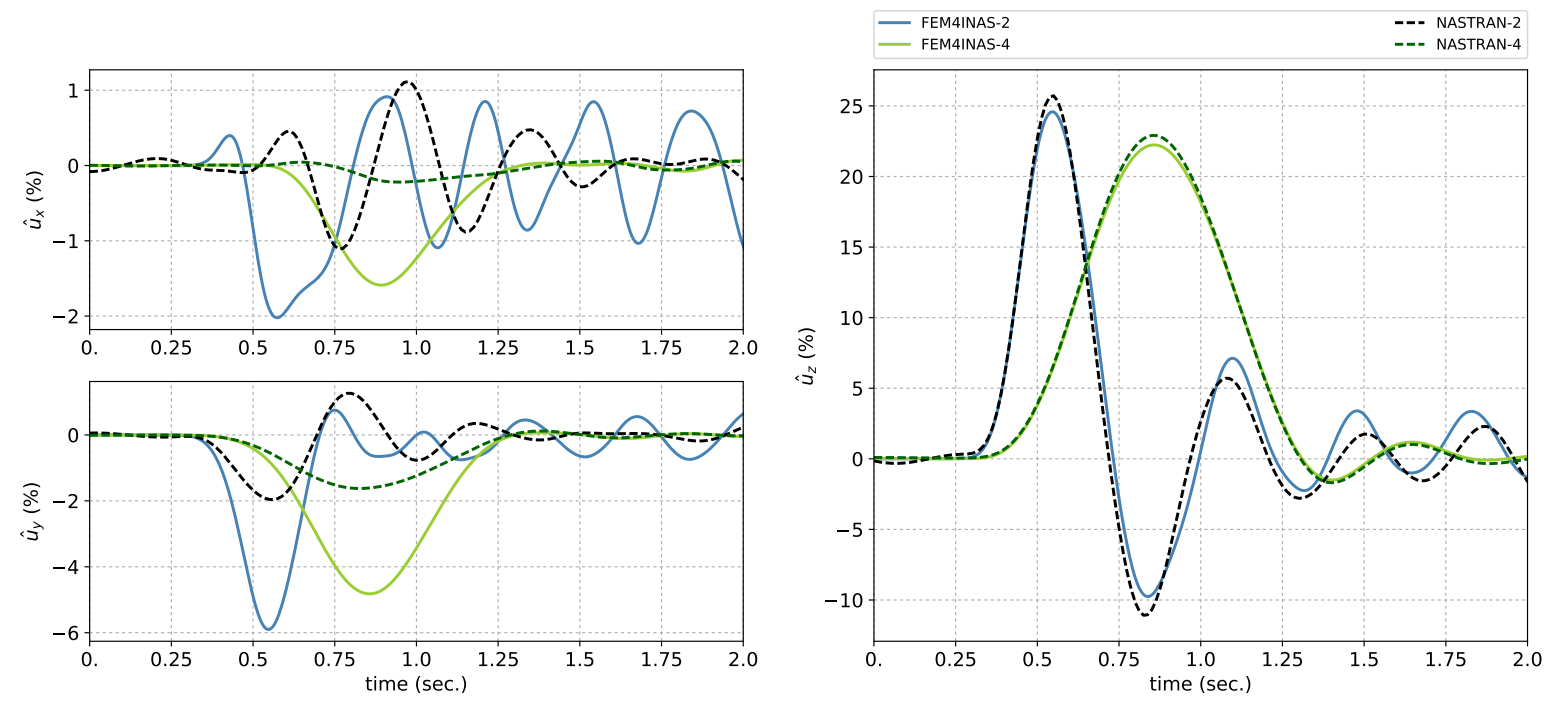

Fig. 13 Wing-tip dynamic response for high intensity gust at Mach 0

The introduction and verification of compressible effects was performed replicating the exercise in Fig. 12 with gust number 3 but including results from a simulation at 0.81 Mach number. A very good match is again found and it is shown in Fig. 14 that the compressible effects act to increase the overall loading on the wings.

Same as before comparisons are presented for large displacements by setting $w_{g 0}=2$ for gust 3 at $M_{\infty}=0$ and $M_{\infty}=0.81$. If compressible and geometrically nonlinear effects are analysed separately, it may be said that the former 

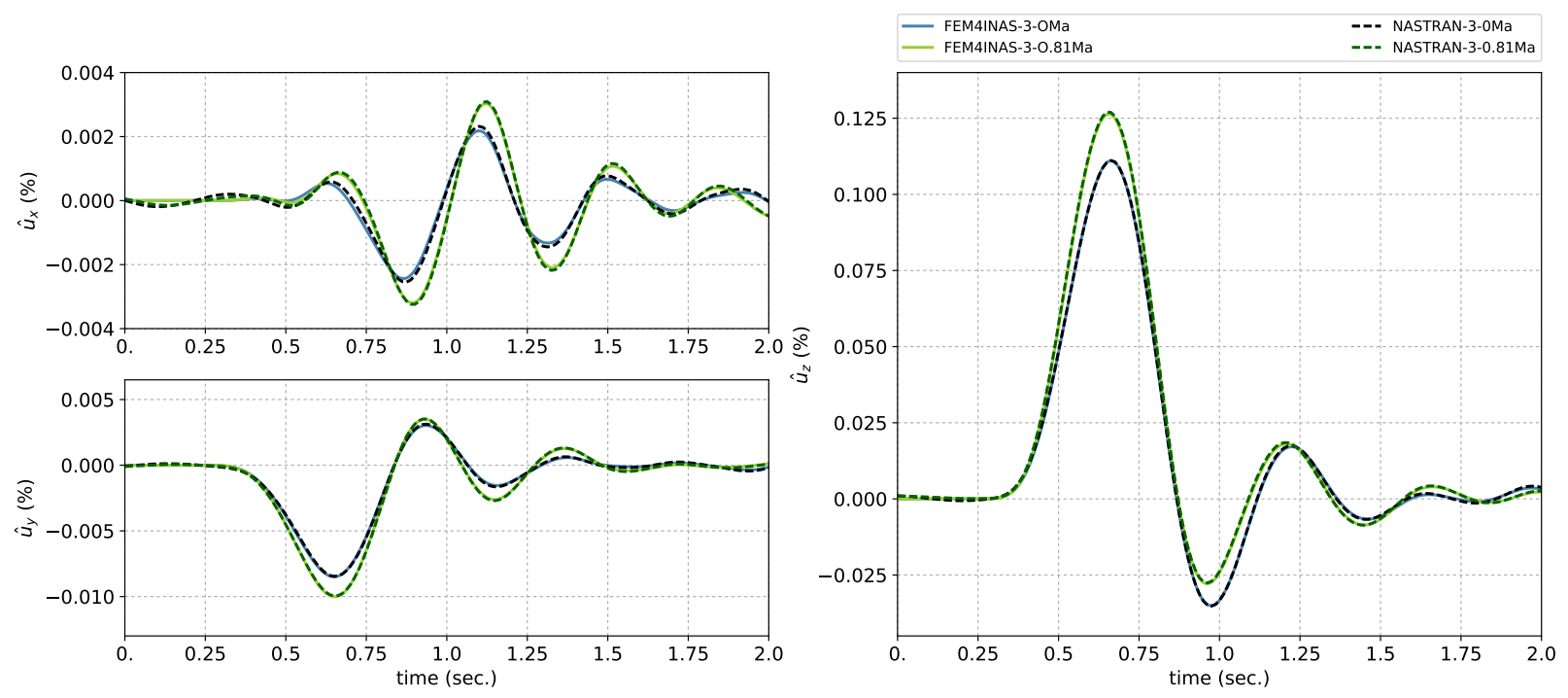

Fig. 14 Wing-tip dynamic response for low intensity gust at Mach 0-0.81

has a more important effect in the z-component of the displacements, i.e. in the module of the aerodynamic loading, while the latter changes the response in $\mathrm{x}$ and $\mathrm{y}$ components more significantly, i.e. in the direction of the loading.
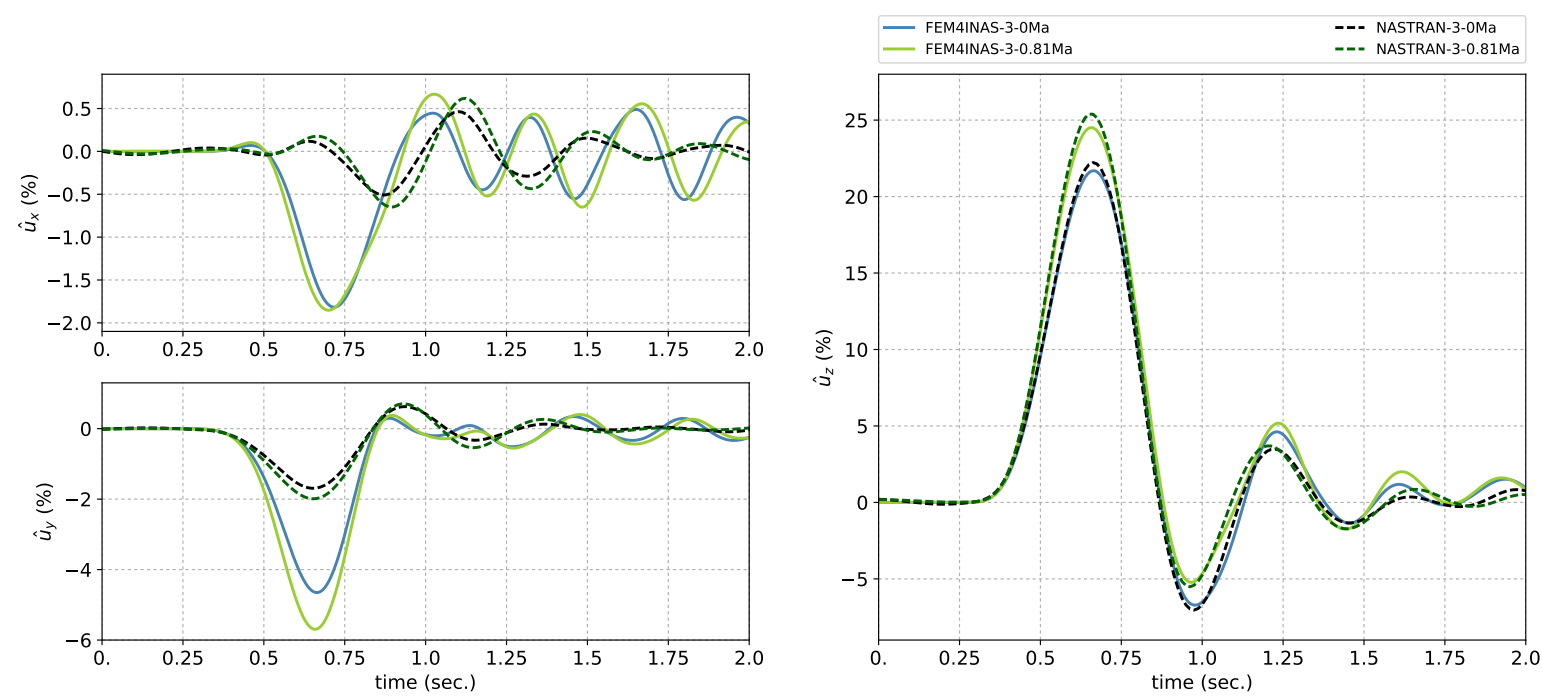

Fig. 15 Wing-tip dynamic response for high intensity gust at Mach 0-0.81

Next we are carrying out simulations with gust shapes (b) and (c) in Fig. 11, gust length is $67 \mathrm{~m}$., air density of $0.778 \mathrm{Kg} / \mathrm{m}^{3}$ and speed of $185 \mathrm{~m} / \mathrm{s}$, and 0 Mach number. The gust intensity is $w_{g 0}=7$ in the asymmetric gust and $w_{g 0}=10$ in the DARPA gust in order to achieve maximum wing tip deformations within $27-33 \%$ in both cases. Instead of plotting those wing displacements, the comparison of moments at the wing root are shown in Fig. 16 as a more relevant metric for design. The plot corresponds to the first second of simulation when the gust acts and maximum loads appear in the structure.

It is worth remarking the moments are are given in a local reference system such as $M_{y}$ is the moment along the wing reference load path, producing torsion of the wing, and $M_{x}$ perpendicular to it and and giving rise to out-of-plane bending deformations . Linear and nonlinear comparisons, both carried out within $\mathrm{FEM}_{4}$ INAS framework, show that linear analysis may produce some non-conservative critical loads, which could lead to failure, or too conservative in other design points, which could mean heavier than necessary structures. This is a known effect in the literature of high 


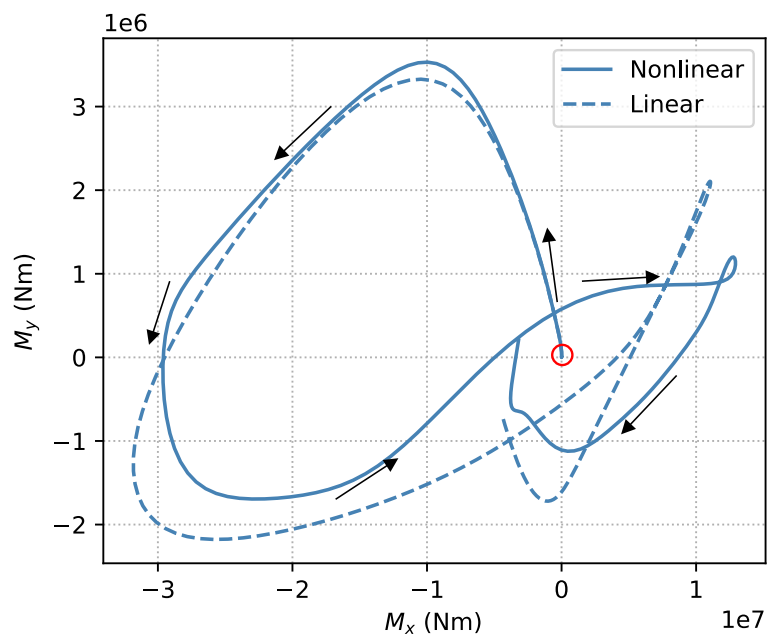

(a) Asymmetric gust shape

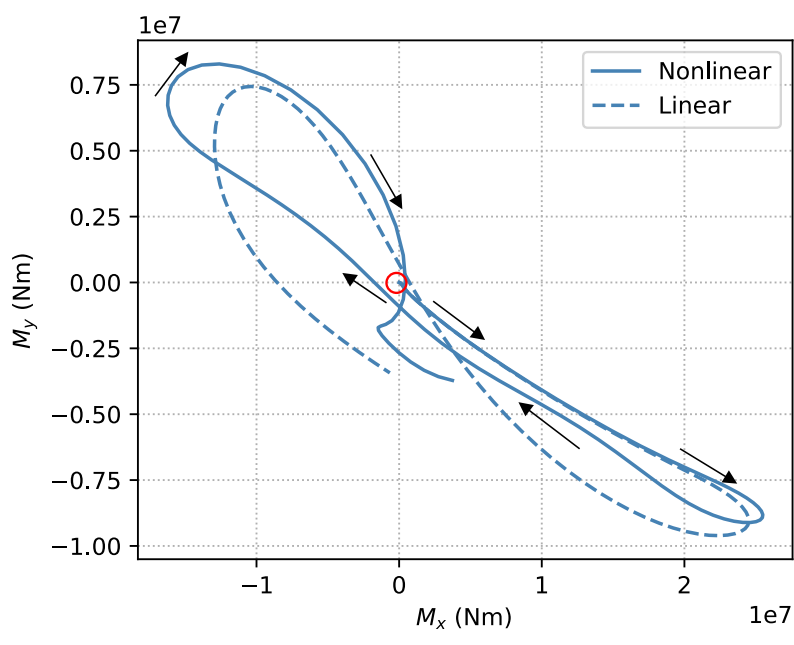

(b) Darpa gust shape

Fig. 16 Wing root moments evolution due to gust disturbance

aspect ratio, long endurance aircraft [1], although it has been rarely explored on large transport aircraft.

\section{E. Nonlinear flutter}

A critical effect in the design of flexible aircraft undergoing large deformations is that of nonlinear flutter [44]: this dynamic instability can change its behaviour when the structural configuration changes due to large deformations, making the flutter point to significantly reduce, which could have drastic consequences to the airframe integrity. Similar to the previous studies, unrealistic angles of attack are used to obtain a proper assessment of the nonlinear effects but the same effects shown here are expected to appear for smaller angles if a less conventional aircraft is studied with very slender and flexible components. Fig. 17] shows the reduction of the flutter speed with AoA and the resultant normalised wing tip deflections just before the instability occurs.

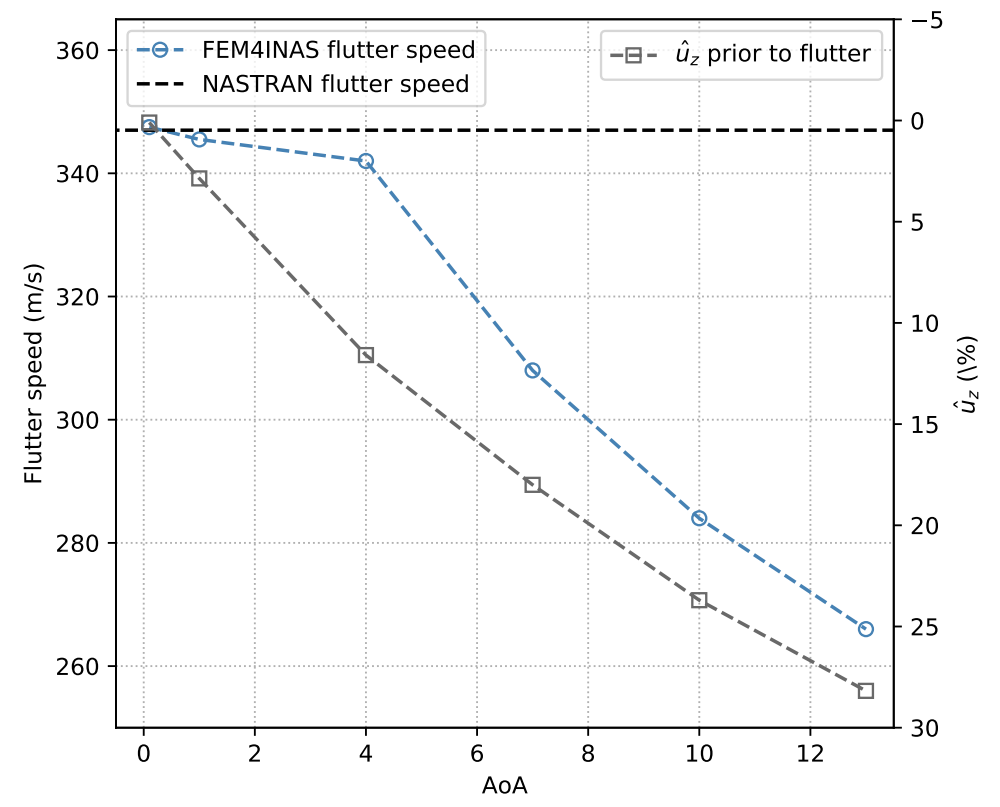

Fig. 17 XRF1 flutter assesment at one design point: $M_{\infty}=0.81$ and $\rho_{\infty}=0.5$ 
The points of study have been $A o A=[0.1,1,4,7,10,13]^{\circ}$, density of air $\rho_{\infty}=0.5 \mathrm{Kg} / \mathrm{m}^{3}$ and Mach number $M_{\infty}=0.81$. While this is an exercise to demonstrate the capabilities of the proposed tool and the effect of nonlinearities in changing stability boundaries, in order to properly assess the flutter envelope of this particular configuration we would have to run many more simulations considering all other combinations of density and Mach number that may be encountered in flight. The linear flutter speed has also been calculated in MSC Nastran using the PK method on the undeformed geometry, and a very good match was obtained with an error of less than $0.3 \%$.

The difference between the linear and nonlinear flutter speed at the $13^{\circ}$ AoA point is of $23.5 \%$. This could have major implications if it was to occur at an AoA of $5^{\circ}$ instead. It is worth remarking that the flutter mechanism is not the same when the equilibrium state is close to the initial configuration or when large deformations have significantly changed the geometry. It has been found that for $A o A=0.1^{\circ}$ oscillations quickly decay or start growing at a rate which is not excessive as shown in Fig. 18(a) At $A o A=10^{\circ}$, on the contrary, oscillations seem to stabilised into the equilibrium position, and all of a sudden grow to infinity in a very sharp manner after the nonlinear flutter velocity, Fig. 18(c) as oppose to Fig. 18(b) where equilibrium is reached.

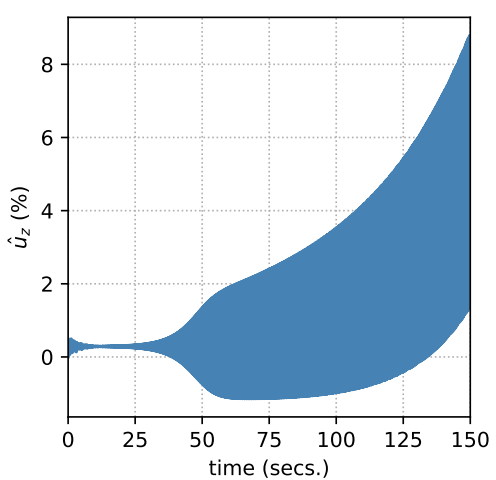

(a) $\mathrm{AoA}=0.1^{\circ}, U_{\infty}=348 \mathrm{~m} / \mathrm{s}$

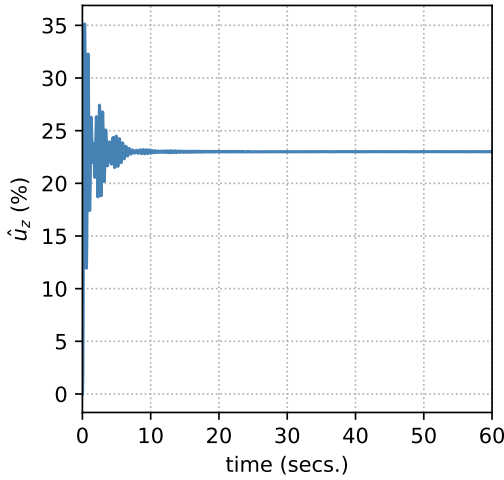

(b) $\mathrm{AoA}=10^{\circ}, U_{\infty}=276 \mathrm{~m} / \mathrm{s}$

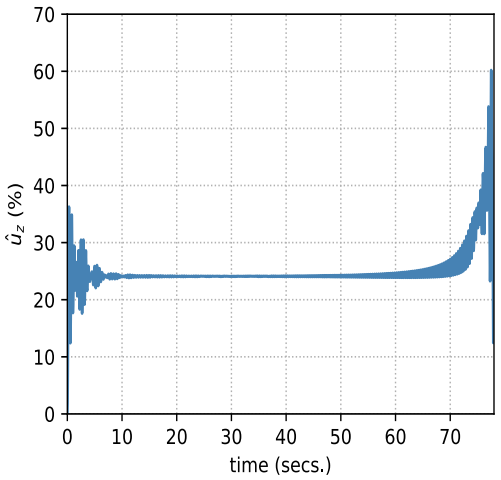

(c) $\mathrm{AoA}=10^{\circ}, U_{\infty}=289 \mathrm{~m} / \mathrm{s}$

Fig. 18 Wing tip deformations for pre and post flutter points. Flutter occurs at $U_{\infty}=347 \mathbf{m} / \mathbf{s}$ and $U_{\infty}=284 \mathbf{m} / \mathbf{s}$ for $A 0 A=0.1^{\circ}$ and $A o A=10^{\circ}$ respectively

\section{Conclusion}

A modal-based description has been presented that seamlessly introduces geometrically-nonlinear effects on generic models built for linear dynamic analysis. The proposed approach preserves the linear solution, which is then augmented with the nonlinear effects in beam theory, namely geometric stiffening, follower forces and changes of inertia properties. To achieve this, firstly, a condensed structure along the main load-path of the full 3D model is obtained using techniques of modal condensation. Secondly, the dynamics of the resulting skeleton-like structure are assumed to be driven by the nonlinear beam intrinsic equations, written in modal space. All the parameters in those equations are obtained through closed-form expressions involving the condensed stiffness and mass matrices, the linear normal modes and, critically, the coordinates of the grid points, which are disregarder in linear theory. Thirdly, influence coefficient matrices, here obtained from the DLM, describe the unsteady aerodynamics in those modal coordinates. This results in a geometrically-nonlinear yet highly computationally efficient aeroelastic system. The three distinctive features of the proposed approach are that: 1) only linear analysis methods are used to manipulate the original 3D model; 2) the equivalent beam model is directly built in modal space with no need to explicitly identify sectional stiffness and inertia; 3) the problem size can be reduced by selecting the number of modes included in the NMROM. Importantly, no model updates are required on either the fluid or the structure (for which only nonlinear quadratic terms are present), which greatly reduces computational time. Therefore, the aeroelastic analysis of future transport aircraft with higher aspect ratio wings might find a good compromise of fidelities in this approach.

Numerical examples have shown the accuracy of the formulation in preserving the characteristics of a full aircraft configuration. Linear aeroelastic calculations in MSC Nastran were in very good agreement with the NMROMs for cases where only small displacements took place. On the other hand the geometrically nonlinear effects were shown for 
trimmed flight under high acceleration loads, manoeuvres at large angles of attack, and dynamic gust disturbances. Furthermore, the nonlinear flutter speed was investigated for increasing variations of angles of attack, which led to a reduction in the flutter speed of up to $23.5 \%$ when compared to the linear solution. Application of the current approach is sought in the realm of future commercial transport aircraft, including designs of radical new configurations, where previous knowledge is very limited, thus a better assessment of in-flight loads may be critical in the success of these aircraft.

Future work will be directed towards improving the accuracy of the aerodynamic loads and assessing further the nonlinear effects coming from the change in the aerodynamic grid. The approach also offers a good platform to couple with a nonlinear controller and evaluate load alleviation techniques in extreme flight conditions.

\section{References}

[1] Cesnik, C. E. S., Palacios, R., and Reichenbach, E. Y., "Re-examined structural design procedures for very flexible aircraft," Journal of Aircraft, Vol. 51, No. 5, 2014, pp. 1581-1591. https://doi.org/10.2514/1.C032464

[2] Noll, T. E., Ishmael, S. D., Henwood, B., Perez-Davis, M. E., Tiffany, G. C., Madura, J., Gaier, M., Brown, J. M., and Wierzbanowski, T., "Technical Findings, Lessons Learned, and Recommendations Resulting from the Helios Prototype Vehicle Mishap," Security, 2007.

[3] Patil, M. J., and Hodges, D. H., "On the importance of aerodynamic and structural geometrical nonlinearities in aeroelastic behavior of high-aspect-ratio wings," Journal of Fluids and Structures, Vol. 19, No. 7, 2004, pp. 905-915. https://doi.org/10. 1016/j.jfluidstructs.2004.04.012, URL www.elsevier.nl/locate/jnlabr/yjfls

[4] Qiao, S., Gao, H., Lyu, Y., Hua, L., and Wang, F., "Nonlinear aeroelastic characteristics analysis of composite wing with high aspect ratio based on co-rotational method," Journal of Fluids and Structures, Vol. 82, 2018, pp. 619-637. https://doi.org/10.1016/j.jfluidstructs.2018.07.009 URL https://doi.org/10.1016/j.jfluidstructs.2018.07.009

[5] Kier, T. M., “An Integrated Model for Lateral Gust Loads Analysis and Dutch Roll Flight Dynamics Using a 3d Panel Method," International Forum on Aeroelasticity and Structural Dynamics, 2017. https://doi.org/10.7150/jca.24838

[6] Wang, Y., Wynn, A., and Palacios, R., "Nonlinear aeroelastic control of very flexible aircraft using model updating," Journal of Aircraft, Vol. 55, No. 4, 2018, pp. 1551-1563. https://doi.org/10.2514/1.C034684

[7] Afonso, F., Vale, J., Oliveira, É., Lau, F., and Suleman, A., "A review on non-linear aeroelasticity of high aspect-ratio wings," Progress in Aerospace Sciences, Vol. 89, 2017, pp. 40-57. https://doi.org/10.1016/j.paerosci.2016.12.004

[8] Dizy, J., Palacios, R., and Pinho, S., "Homogenisation of slender periodic composite structures," International Journal of Solids and Structures, Vol. 50, No. 9, 2013, pp. 1473-1481. https://doi.org/10.1016/j.ijsolstr.2013.01.017.

[9] Elsayed, M. S. A., Sedaghati, R., and Abdo, M., "Accurate Stick Model Development for Static Analysis of Complex Aircraft Wing-Box Structures,” AIAA Journal, Vol. 47, No. 9, 2009, pp. 2063-2075. https://doi.org/10.2514/1.38447

[10] Riso, C., Sanghi, D., Cesnik, C. E., Vetrano, F., and Teufel, P., "Parametric Roll Maneuverability Analysis of a High-AspectRatio-Wing Civil Transport Aircraft," AIAA Scitech 2020 Forum, American Institute of Aeronautics and Astronautics, 2020. https://doi.org/10.2514/6.2020-1191. URL https://doi.org/10.2514/6.2020-1191

[11] Cea, A., and Palacios, R., "Nonlinear Modal Aeroelastic Analysis from Large Industrial-Scale Models," AIAA Scitech 2019 Forum, American Institute of Aeronautics and Astronautics, 2019. https://doi.org/10.2514/6.2019-0208.

[12] Medeiros, R. R., Cesnik, C. E. S., and Coetzee, E. B., "Computational Aeroelasticity Using Modal-Based Structural Nonlinear Analysis," AIAA Journal, 2019, pp. 1-10. https://doi.org/10.2514/1.j058593. URL https://doi.org/10.2514/1.J058593

[13] Mignolet, M. P., Przekop, A., Rizzi, S. A., and Spottswood, S. M., "A review of indirect/non-intrusive reduced order modeling of nonlinear geometric structures," Journal of Sound and Vibration, Vol. 332, No. 10, 2013, pp. 2437-2460. https://doi.org/10.1016/j.jsv.2012.10.017

[14] Drachinsky, A., and Raveh, D. E., "Modal rotations: A modal-based method for large structural deformations of slender bodies," AIAA Journal, Vol. 58, No. 7, 2020, pp. 3159-3173. https://doi.org/10.2514/1.J058899

[15] Wang, Y., Palacios, R., and Wynn, A., "A method for normal-mode-based model reduction in nonlinear dynamics of slender structures," Computers and Structures, Vol. 159, 2015, pp. 26-40. https://doi.org/10.1016/j.compstruc.2015.07.001. 
[16] Cea, A., and Palacios, R., "A Non-Intrusive Geometrically Nonlinear Augmentation to Generic Linear Aeroelastic Models (Accepted for publication)," Journal of Fluids and Structures, 2020.

[17] Hodges, D. H., "Geometrically Exact, Intrinsic Theory for Dynamics of Curved and Twisted Anisotropic Beams," AIAA Journal, Vol. 41, No. 6, 2003, pp. 1131-1137. https://doi.org/10.2514/2.2054

[18] Modaress-Aval, A. H., Bakhtiari-Nejad, F., Dowell, E. H., Peters, D. A., and Shahverdi, H., "A Comparative Study of Nonlinear Aeroelastic Models for High Aspect Ratio Wings," Journal of Fluids and Structures, Vol. 85, 2019, pp. $249-274$. https://doi.org/10.1016/j.jfluidstructs.2019.01.003

[19] Albano, E., and Rodden, W. P., "A doublet-lattice method for calculating lift distributions on oscillating surfaces in subsonic flows.” AIAA Journal, Vol. 7, No. 2, 1969, pp. 279-285. https://doi.org/10.2514/3.5086

[20] Rodden, W. P., Theoretical and computational aeroelasticity, Crest Publishing, California, USA, 2011.

[21] Kim, T., "Flutter Prediction Methodology Based on Dynamic Eigen Decomposition and Frequency-Domain Stability," Journal of Fluids and Structures, Vol. 86, 2019, pp. 354 - 367. https://doi.org/10.1016/j.jfluidstructs.2019.01.022.

[22] Murua, J., Palacios, R., and Graham, J. M. R., "Assessment of wake-tail interference effects on the dynamics of flexible aircraft," AIAA Journal, Vol. 50, 2012, pp. 1575-1585. https://doi.org/10.2514/1.J051543

[23] Palacios, R., Climent, H., Karlsson, A., and Winzell, B., "Assessment of Strategies for Correcting Linear Unsteady Aerodynamics Using CFD or Experimental Results," Progress in Computational Flow-Structure Interaction, Springer Verlag, 2003, Chap. 8, pp. 209-224.

[24] Valente, C., Wales, C., Jones, D., Gaitonde, A., Cooper, J. E., and Lemmens, Y., "An optimized doublet-lattice method correction approach for a large civil aircraft," 17th International Forum on Aeroelasticity and Structural Dynamics, IFASD 2017, Vol. 2017-June, No. June, 2017, pp. 1-15.

[25] Güner, H., Thomas, D., Dimitriadis, G., and Terrapon, V. E., "Unsteady aerodynamic modeling methodology based on dynamic mode interpolation for transonic flutter calculations," Journal of Fluids and Structures, Vol. 84, 2019, pp. $218-232$. https://doi.org/10.1016/j.jfluidstructs.2018.11.002 URL https://doi.org/10.1016/j.jfluidstructs.2018.11.002

[26] Ripepi, M., Verveld, M. J., Karcher, N. W., Franz, T., Abu-Zurayk, M., Görtz, S., and Kier, T. M., "Reduced-order models for aerodynamic applications, loads and MDO," CEAS Aeronautical Journal, Vol. 9, No. 1, 2018, pp. 171-193. https://doi.org/10.1007/s13272-018-0283-6

[27] Roger, K. L., “Airplane Math Modeling Methods for Active Control Design," Structural Aspects of Active Controls, AGARD CP-228, 1977, pp. 4.1-4.11.

[28] Palacios, R., "Invariant Manifolds in Beam Dynamics: Free Vibrations and Nonlinear Normal Modes," Encyclopedia of Continuum Mechanics, Springer Berlin Heidelberg, 2017, pp. 1-8. https://doi.org/10.1007/978-3-662-53605-6_56-1

[29] Yagil, L., Raveh, D. E., and Idan, M., "Deformation control of highly flexible aircraft in trimmed flight and gust encounter," Journal of Aircraft, Vol. 55, 2018, pp. 829-840. https://doi.org/10.2514/1.C034353, URL http://arc.aiaa.org

[30] Castrichini, A., Hodigere Siddaramaiah, V., Calderon, D. E., Cooper, J. E., Wilson, T., and Lemmens, Y., "Nonlinear Folding Wing Tips for Gust Loads Alleviation," Journal of Aircraft, Vol. 53, No. 5, 2016, pp. 1391-1399. https://doi.org/10.2514/1.C033474

[31] Klimmek, T., "Parametric Set-Up of a Structural Model for FERMAT Configuration for Aeroelastic and Loads Analysis," ASD Journal, Vol. 3, No. 2, 2014, pp. 31-49. https://doi.org/10.3293/asdj.2014.27

[32] Palacios, R., Murua, J., and Cook, R., "Structural and aerodynamic models in nonlinear flight dynamics of very flexible aircraft," AIAA Journal, Vol. 48, No. 11, 2010, pp. 2648-2659. https://doi.org/10.2514/1.J050513.

[33] Palacios, R., "Nonlinear normal modes in an intrinsic theory of anisotropic beams," Journal of Sound and Vibration, Vol. 330, No. 8, 2011, pp. 1772-1792. https://doi.org/10.1016/j.jsv.2010.10.023

[34] Karpel, M., "Design for Active Flutter Suppression and Gust Alleviation Using State-Space Aeroelastic Modeling," Journal of Aircraft, Vol. 19, No. 3, 1982, pp. 221-227. https://doi.org/10.2514/3.57379

[35] Botez, R. M., and Biskri, D. E., "Unsteady Aerodynamic Forces Mixed Method for Aeroservoelasticity Studies on an F/A-18 Aircraft," Journal of Aircraft, Vol. 44, No. 4, 2007, pp. 1378-1383. https://doi.org/10.2514/1.19329. 
[36] Dinu, A. D., Botez, R. M., and Cotoi, I., "Aerodynamic Forces Approximations Using the Chebyshev Method for ClosedLoop Aeroservoelasticity Studies," Canadian Aeronautics and Space Journal, Vol. 51, No. 4, 2005, pp. $167-175$. https://doi.org/10.5589/q05-016.

[37] Baker, M. L., Goggin, P. J., and Winther, B. A., "DTIC ADP010476: Aeroservoelastic Modeling, Analysis, and Design Techniques for Transport Aircraft," RTO MP-36: Structural Aspects of Flexible Aircraft Control, 1999.

[38] Tiffany, S. H., and Adams, W. M., "Nonlinear Programming - Extensions to Rational Function Approximation Methods for Unsteady Aerodynamic Forces Nonlinear Programming Extensions to Rational Function Approximation Methods for Unsteady Aerodynamic Forces," NASA Technical Paper, 1988, p. 56.

[39] Eversman, W., and Tewari, A., "Consistent Rational-Function Approximation for Unsteady Aerodynamics," Journal of Aircraft, Vol. 28, No. 9, 1991, pp. 545-552. https://doi.org/10.2514/3.46062

[40] Kier, T. M., Looye, G. H. N., and Looye, G. H. N., "Unifying manoeuvre and gust loads analysis models," International Forum of Aeroelasticity and Structural Dynamics (IFASD) 2009, 2009, pp. 1-20.

[41] Kier, T. M., “An Integrated Loads Analysis Model for Wake Vortex Encounters," International Forum on Aeroelasticity and Structural Dynamics, 2013, pp. 1-16.

[42] Wright, J. R., and Cooper, J. E., Introduction to Aircraft Aeroelasticity and Loads, $2^{\text {nd }}$ ed., John Wiley \& Sons Ltd, West Sussex, UK, 2007.

[43] Palacios, R., and Cea, A., "Nonlinear Modal Condensation of Large Finite-Element Models: An Application of Hodges' Intrinsic Theory," AIAA Journal, 2018.

[44] Su, W., and Cesnik, C. E. S., "Nonlinear aeroelasticity of a very flexible blended-wing-body aircraft," Journal of Aircraft, Vol. 47, No. 5, 2010, pp. 1539-1553. https://doi.org/10.2514/1.47317 